\title{
Low-level laser therapy in temporomandibular joint disorders: a systematic review
}

Syed Ansar Ahmad ${ }^{1}$, Shamimul Hasan ${ }^{2 *}$, Shazina Saeed ${ }^{3}$, Ateeba Khan ${ }^{4}$, Munna Khan ${ }^{5}$

\author{
Author Affiliations: \\ 1. Department of Oral Surgery, Faculty of Dentistry, Jamia Millia Islamia, New Delhi, India \\ 2. Department of Oral Medicine and Radiology, Faculty of Dentistry, Jamia Millia Islamia, \\ New Delhi, India \\ 3. Laboratory of Disease Dynamics and Molecular Epidemiology, \\ Amity Institute of Public Health, Amity university, Noida, Uttar Pradesh, India \\ 4. Faculty of Dentistry, Jamia Millia Islamia, New Delhi, India \\ 5. Department of Electrical Engineering, Jamia Millia Islamia, New Delhi, India
}

\author{
* Corresponding Author: \\ Dr. Shamimul Hasan, Professor, \\ Department of Oral Medicine \\ and Radiology, Faculty of \\ Dentistry, Jamia Millia Islamia, \\ New Delhi, India. \\ Phone: 9953290676 \\ E-mail: shasan1@jmi.ac.in \\ DOI \\ 10.25122/jml-2020-0169 \\ Dates \\ Received: 1 November 2020 \\ Accepted: 29 March 2021
}

\begin{abstract}
Temporomandibular joint disorders (TMDs) encompass a wide array of ailments affecting the temporomandibular joint (TMJ), muscles of mastication, and the allied structural framework. Myofascial pain, internal derangement of the joint, and degenerative joint diseases constitute the majority of TMDs. TMDs usually have a multifactorial etiology, and treatment modalities range from conservative therapies to surgical interventions. Low-level laser therapy (LLLT) has evolved as an efficient non-invasive therapeutic modality in TMDs. Previously conducted systematic reviews and meta-analyses have shown variable results regarding the efficiency of LLLT in TMJ disorder patients. Hence, this systematic review was carried out as an attempt to evaluate the efficacy of LLLT in the treatment of temporomandibular joint disorder patients.
\end{abstract}

KEYWORDS: low-level laser therapy (LLLT), pain intensity, randomized controlled trials (RCTs), temporomandibular joint disorders (TMDs).

\section{INTRODUCTION}

TMJ disorders (TMDs) are categorized as degenerative musculoskeletal disorders causing structural and functional abnormalities [1]. Pain, diminished jaw functions and movements, midline deviation, malocclusion, joint noises, and locking constitutes the cardinal signs and symptoms of TMDs $[2,3]$. The overall incidence of TMDs ranges from $21.5 \%$ to $50.5 \%$, with a female gender predilection [4]. TMDs are categorized into three forms. Myofascial pain is the most typical form, followed by internal derangement of the joint and degenerative joint disease, respectively [5]. TMDs represent a primary cause of non-odontogenic pain in the orofacial region, with $40-75 \%$ of the individuals showing at least one TMD sign, such as TMJ noise, and 33\% at least one symptom, TMJ or facial pain [6]. Many TMDs may be self-limiting, with periodic remission and exacerbation of symptoms [7].

TMD therapies primarily aim to eliminate pain, joint clicking, restoring TMJ functions and entails dietary and behavioral amendments, pharmacotherapy, physical therapy, occlusal splint therapy, intra-articular injections, arthroscopy, arthrocentesis, Lasers, or open joint surgery [8]. Lasers have gained wide applications in dentistry owing to their therapeutic attributes, such as tissue healing and enhanced local microcirculation [9]. Low-level laser therapy (LLLT) refers to a light-based therapy that produces monochromatic and coherent light of a single wavelength [3]. 


\section{JOURNAL of MEDICINE and LIFE}

LLLT may act via numerous mechanisms of action, including facilitating the release of endogenous opioids, augmenting tissue repair and cellular respiration, increasing vasodilatation and pain threshold, and decreasing inflammation [10]. LLLT exerts a photochemical effect, in contrast to the ablative or thermal effects related to medical laser procedures [11].

The current state of knowledge in LLLT as a therapeutic modality in TMDs is primarily based upon previously conducted prospective clinical trials, which have yielded debatable outcomes [12-16]. Few studies have demonstrated higher efficacy of LLLT over placebo $[12,15,16]$, while others have shown similar efficiency of LLLT and placebo in the treatment of TMD [13, 14].

Many systematic reviews with or without meta-analyses have also demonstrated contentious results regarding the effectiveness of LLLT in TMDs [17-19]. A systematic review by Melis et al. demonstrated better efficacy of LLLT in eliminating TMJ pain as compared to the masticatory muscle diseases [20]. The meta-analyses by Gam et al. [21], Petrucci et al. [18], and McNeely et al. [22] could not establish the efficacy of LLLT therapy in TMJ pain. However, a meta-analysis conducted by Chang et al. suggested that LLLT has a reasonable analgesic effect on TMJ pain [19]. A meta-analysis by Chen et al. reported that LLLT might substantially enhance the functional outcomes with limited pain amelioration in TMD patients [23]. A systematic review with meta-analyses demonstrated that LLLT is not only effective in pain relief but also improves functional outcomes in TMD patients [4]. Few randomized controlled trials (RCTs) documenting the efficacy of LLLT in TMDs have been conducted since the last published systematic review [5, 11, 24-27].

However, to date, there is still no conclusive validation to substantiate or contradict LLLT for TMDs. Hence, this systematic review was conducted to substantiate and re-validate the efficacy of LLLT as a therapeutic modality in TMDs and review the evidence from previously published literature. The study results are also expected to serve as useful insight and guidelines for clinical practitioners treating patients with TMDs. This review will provide precise and obvious knowledge about the benefits and procedures of laser application, which have already been successfully established in TMD management.

Our objectives were to:

- Ascertain the efficacy of LLLT in pain diminution as the primary outcome and secondary outcome on TMJ functions, masticatory efficiency, psychological and emotional aspects;

- $\quad$ Compare LLLT with placebo and other interventions used in TMD management.

\section{MATERIAL AND METHODS}

A systematic literature review was carried out to assess the efficiency of low-level laser therapy in patients with temporomandibular joint disorders.

\section{Research questions}

The search for the systematic review was initiated by defining the keywords concerning the population, intervention, control, and outcomes (PICO) format: a) population - "temporomandibular joint disorders (TMDs)"; b) intervention/exposure - "low-level laser therapy (LLLT)"; c) control - "placebo or other interventions like occlusal splints, analgesics, transcutaneous electrical nerve stimulation (TENS) and botulinum toxins"; and d) outcome - "efficacy assessment". The research question was designed for the above-mentioned keywords: a) "Is low-level laser therapy (LLLT) efficacious in patients with temporomandibular joint disorders"?

\section{Literature search and identification of studies}

This search strategy followed the Cochrane guidelines for a systemic review. An extensive hand-searching and electronic searching were made between January 2000 to June 2020 using the combination of controlled vocabulary and free text terms in PubMed and Science direct search engines.

\section{Inclusion criteria}

a) RCTs involving LLLT therapy in human subjects with TMDs; b) articles published in the English language between January 2000 to June 2020; c) at least a total of 10 study subjects (both LLLT and placebo categories).

\section{Exclusion criteria}

a) Nonrandomized or crossover studies (studies other than RCTs); b) studies conducted on animal models; c) articles published in languages other than English and before January 2000; d) study subjects less than 10; e) studies that fail to provide information on the 


\section{JOURNAL of MEDICINE and LIFE}

outcomes of interest and f) subjects with systemic disorders (i.e., rheumatoid arthritis and fibromyalgia) or non-TMD related pain (i.e., odontogenic pain, neuralgia, and psychological dysfunctions).

\section{Study selection}

The titles and abstracts of the identified studies were thoroughly evaluated for potential eligibility. Studies that did not assess the efficacy of LLLT on TMDs were excluded. However, if the abstract of the study was unclear, the full texts of the study were then procured for evaluation. Manual cross-referencing of all the retrieved articles was carried out to identify any study missed previously.

\section{Outcome parameters}

The primary outcome parameter was a diminution in the pain intensity in TMDs after LLLT therapy, expressed by the visual analog scale (VAS). The secondary outcome parameters were the effect on TMJ functions (expressed in terms of mouth opening, lateral and protrusive mandibular excursive movements, and TMJ noises), masticatory efficiency, pressure pain threshold (PPT), electromyographic (EMG) activity, quality of life (QoL), psychological and emotional aspects associated with TMDs.

\section{Data extraction}

Data extraction was made based on the first author, year of publication, journal name, sample size, treatment design, type and wavelength of laser, dose and power of the used laser, study design, study outcome, and results. The included studies were reviewed by two other authors.

\section{Risk of bias assessment}

The risk of publication bias was assessed by using the R-based Robvis software package introduced by the National Institute for Health Research (NIHR) (https://www.riskofbias.info/welcome/robvis-visualization-tool).

\section{RESULTS}

Thirty-seven articles were considered eligible for this systematic review. The selection cycle is in accordance with the Preferred Reporting Items for Systematic Reviews and Meta-Analyses (PRISMA) guidelines and is represented as a flowchart in Figure 1.

Based on visual inspection of the figure generated by the Robvis software package, there is no potential publication bias in this study assessing the effectiveness of low-level laser treatment used in various RCTs for TMD patients (Figures 2 and 3). Out of 37 studies, 33 $(89.18 \%)$ are high methodological studies, which have an overall low risk of bias or with some concerns, while only 4 studies have a high risk of bias. A detailed description of the eligible studies is given in Tables 1 and 2.

\section{Characteristics of the studies}

Eighteen studies used the "Research Diagnostic Criteria" (RDC/TMD) for diagnosis of TMDs, followed by VAS in 6 conducted RCTs. 7 studies utilized a combination of these two diagnostic criteria. A wide variety of lasers were used in the included studies. Nineteen studies used a Gallium-aluminum-arsenide laser (GaAlAs). Gallium-arsenide laser (GaAs) was used in 5 studies. Neodymium-doped yttrium aluminum garnet (Nd: YAG), diode lasers, and red and infrared lasers were applied in 2 studies each, followed by Indium-gallium-aluminum-phosphide laser (InGaAlP) and Helium-neon laser $(\mathrm{HeNe})$, which were used in one study each as shown in Table 1. A combination of two laser types was also used in 3 studies, namely that of Shirani et al. [28], Demirkol et al. [29], and Pereira et al. [30]. A single laser type at two different wavelengths (GaAlAs at $650 \mathrm{~nm} / 830 \mathrm{~nm}$ ) was used in an RCT by Wang et al. [16]. Single laser with two or three laser dosages was employed in 4 studies (Table 1).

The shortest and longest laser wavelengths used among the included studies were $632.8 \mathrm{~nm}$ [13] and $1064 \mathrm{~nm}$ [29, 31], respectively, except for Altindis et al. [25] and Rodriguez et al. [27], who did not mention the wavelength used in their lasers therapy. Laser dosage ranged between $1.5 \mathrm{~J} / \mathrm{cm} 2$ to $112.5 \mathrm{~J} / \mathrm{cm} 2$ for the majority of the studies. Laser power ranged between $1.76 \mathrm{Mw}$ [32] to 500mW [33]; 3 studies did not mention the power of the laser [25, 27,30]. Temporomandibular joint and/or the affected muscles were the primary site of laser application in 18 of the conducted RCTs. Laser therapy was applied specifically at the TMJ in 9 RCTs. In 8 RCTs, the site of laser application was only in the muscles. In most of the conducted studies, laser application was made at pre-decided sites, irrespective of the fact that they were the points of maximum pain or not. However, in other RCTs, only the points of maximum pain intensity were irradiated (Table 2). 


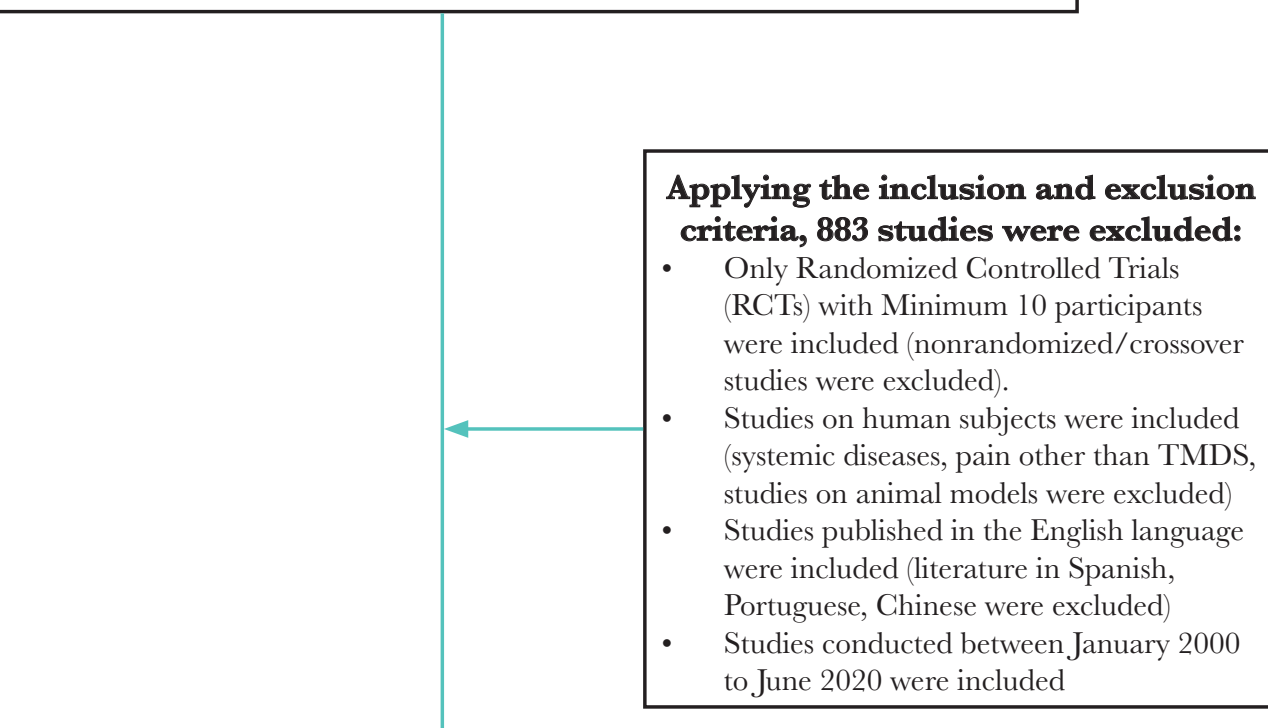

Total 164 studies included for further assessment

38 studies identified from PubMed

126 studies identified from Science Direct

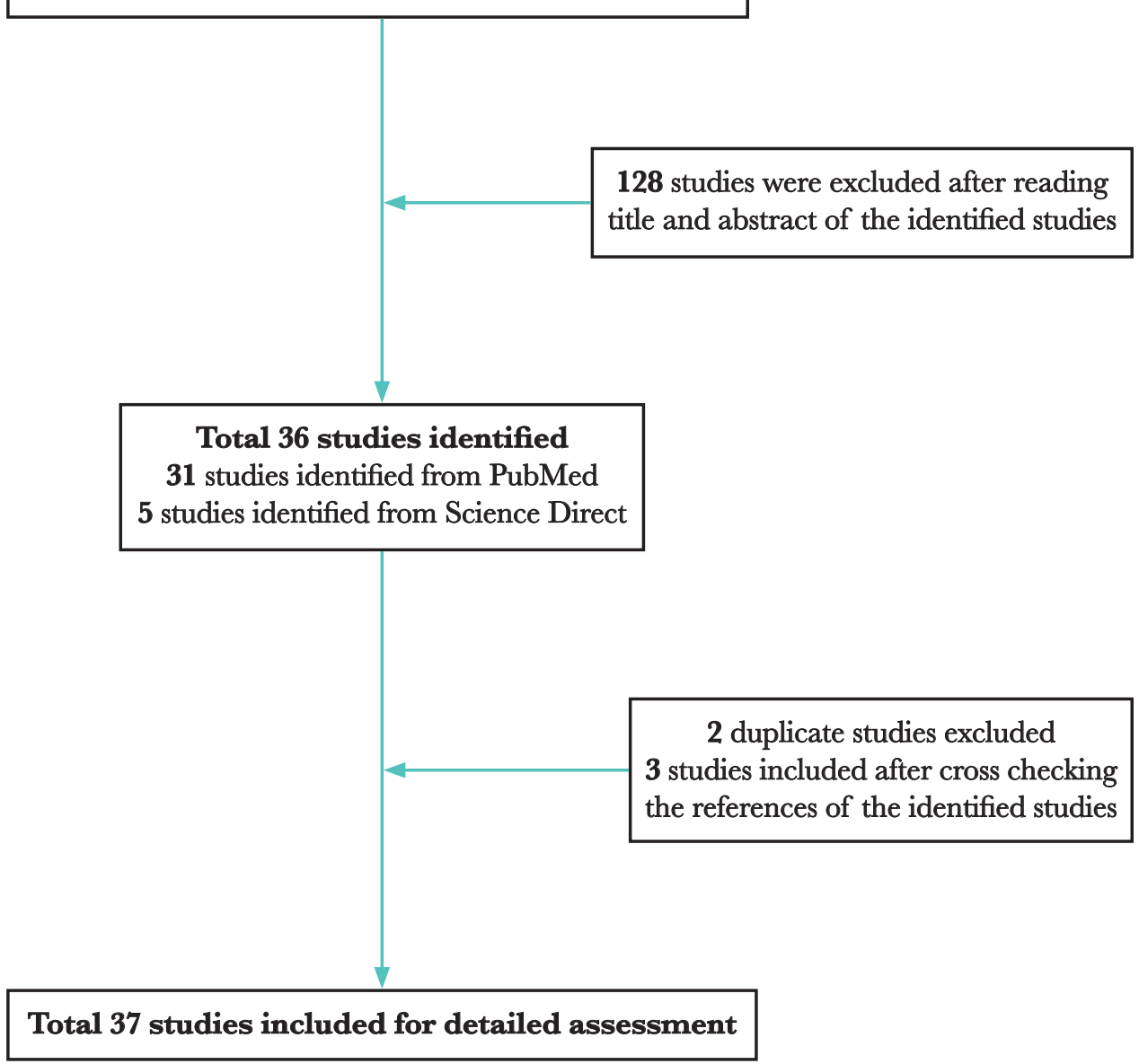




\section{JOURNAL of MEDICINE and LIFE}

Bias arising from the randomization process Bias due to deviations from intended interventions Bias due to missing outcome data Bias in measurement of the outcome Bias in selection of the reported result Overall risk of bias

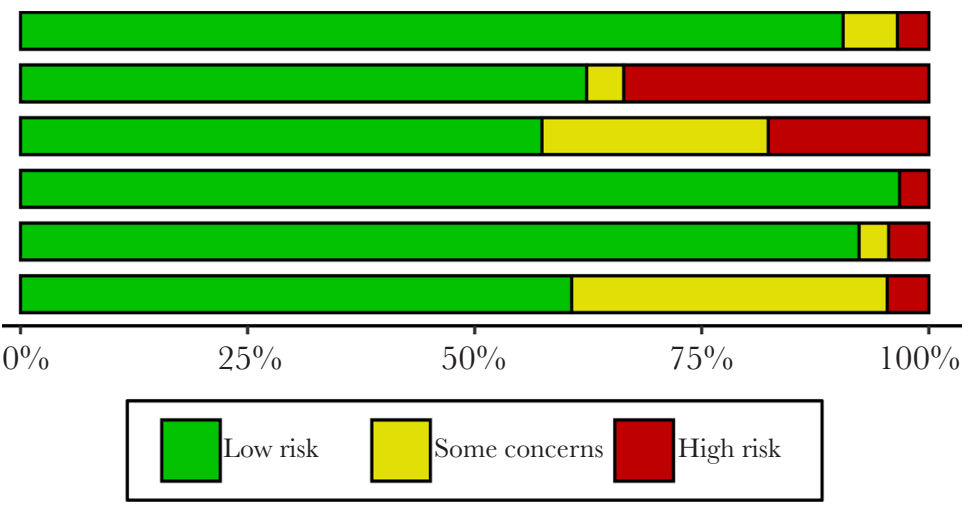

Figure 2. Robvis output for risk bias assessment.

Most of the studies involved a comparison of LLLT and placebo groups. However, seven studies involved comparison of laser with other interventions, namely, botulinum toxin A [9], TENS therapy [11], ibuprofen [15], needling [34], occlusal splints [33, 35], physiotherapeutic and drug protocol (PDP) [36]. Two studies incorporated co-interventions equally to both LLLT and placebo groups. Piroxicam was incorporated with LLLT in one study [37], and in the other study, oral motor (OM) exercises were combined with LLLT [38].

Most of the included studies provided data on the primary outcome of laser therapy, like pain intensity. Eighteen studies focused on secondary outcomes like mouth opening (MO), followed by 13 studies on lateral excursive (LE) mandibular movements, 10 studies on protrusive excursive (PE) mandibular movements, 7 studies on PPT, and 2 studies each on EMG, joint noises, TMD related psychological and emotional aspects, masticatory efficiency (ME), respectively. One study each focused on subjective tinnitus and occlusal contacts distribution (Table 1).

Eighteen studies showed that LLLT was efficacious in diminishing TMD pain, whereas 12 studies showed that LLLT had similar efficacy as of placebo/controls/other intervention in TMD pain diminution. Four studies presented varied effects of LLLT on pain intensity, mandibular motion, EMG activity, and masticatory efficiency. Two studies revealed that LLLT improved the psychological and emotional aspects associated with TMDs, joint noises, masticatory efficiency, and EMG parameters, respectively. One study focused on subjective tinnitus, whereas another study suggested laser acupuncture (LAT) therapy as a suitable alternative to LLLT. The results demonstrate that LLLT appears to be efficient in diminishing TMD pain with variable effects on the outcome of secondary parameters (Table 1).

\section{DISCUSSION}

Orofacial pain/pain in the stomatognathic system region has a varied pathophysiological basis, and its diagnosis and therapy cover diverse aspects of medicine and dentistry. TMDs are one of the principal causes of orofacial pain. According to the International Association for the Study of Pain, TMDs are defined as an assembly of painful musculoskeletal disorders of the temporomandibular joints, masticatory muscles, and adjacent architecture [39].

The exact etiology of TMDs is still not completely elucidated; however, stress-induced repetitive jaw clenching and grinding accounts as the most important causative factor. Stress also plays a major role in sustaining and augmenting the TMD symptoms. TMDs pose significant diagnostic and therapeutic challenges owing to their multifactorial etiology, lack of investigative guidelines and strategies, and are widely considered as a physical, psychological, and functional disorder [40].

A vast majority of studies assessing TMD therapeutic protocols incorporate only pain scales (VAS) and MO analysis, thereby omitting other imperative characteristics like chronic pain, stress, anxiety, and depression. Dworkin and Le Resche later adopted the Research Diagnostic Criteria (RDC/TMD) in 1992 to overpower these discrepancies, and it also provided the academicians and practitioners with an effective and systematic method of examination, diagnosis, and classification of TMDs [24].

In our systematic review, 18 studies used RDC/TMD to diagnose TMDs. Six RCTs utilized VAS, whereas 7 studies utilized a combination of these two diagnostic criteria. TMDs generally have a gender predisposition, the disease predominantly affecting females (F:M $=2: 1-8: 1)$. Patients in the age group of 20 and 50 years are usually affected, an unusual age distribution for a degenerative disorder [1]. In our systematic review, most of the studies revealed a higher prevalence of TMDs among women compared to men with an age range between 20-55 years. Pain is the cardinal manifestation in TMDs. Pain in TMDs accounts for the most probable explanation of these patients seeking treatment. This also serves as a justification for most of the studies focused on assessing the efficacy of a wide 
JOURNAL of MEDICINE and LIFE

Risk of bias domains

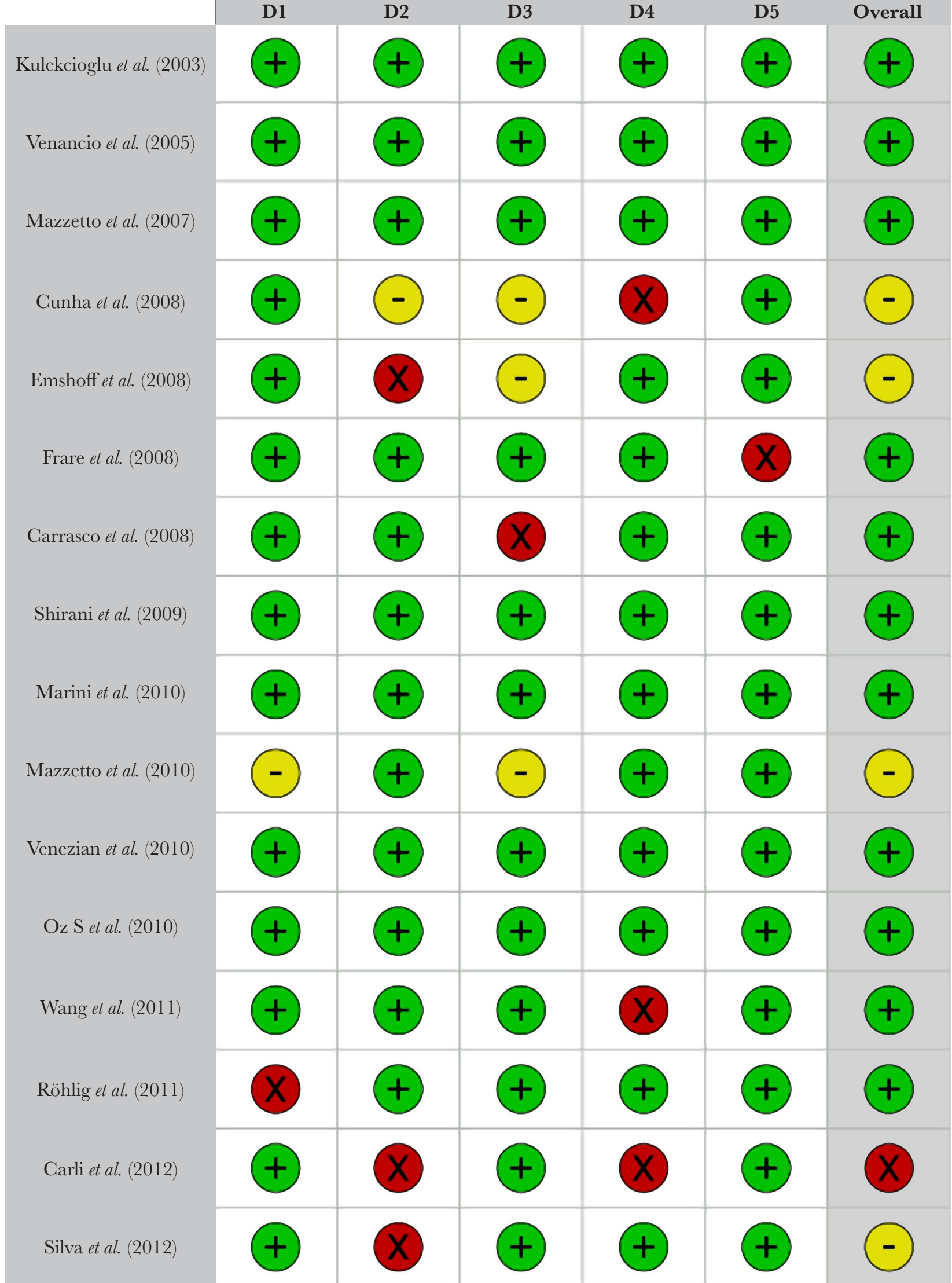


JOURNAL of MEDICINE and LIFE

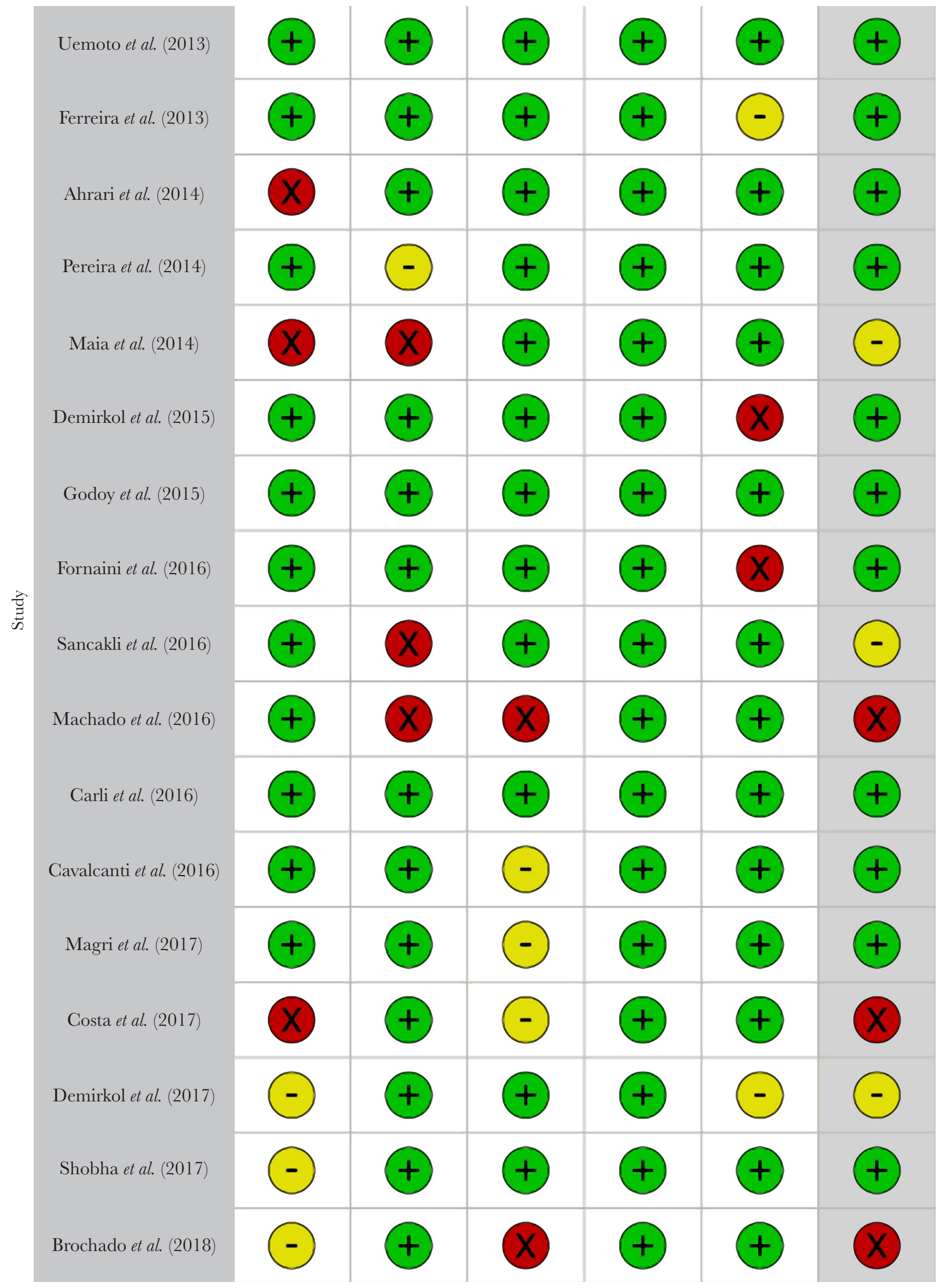




\section{JOURNAL of MEDICINE and LIFE}

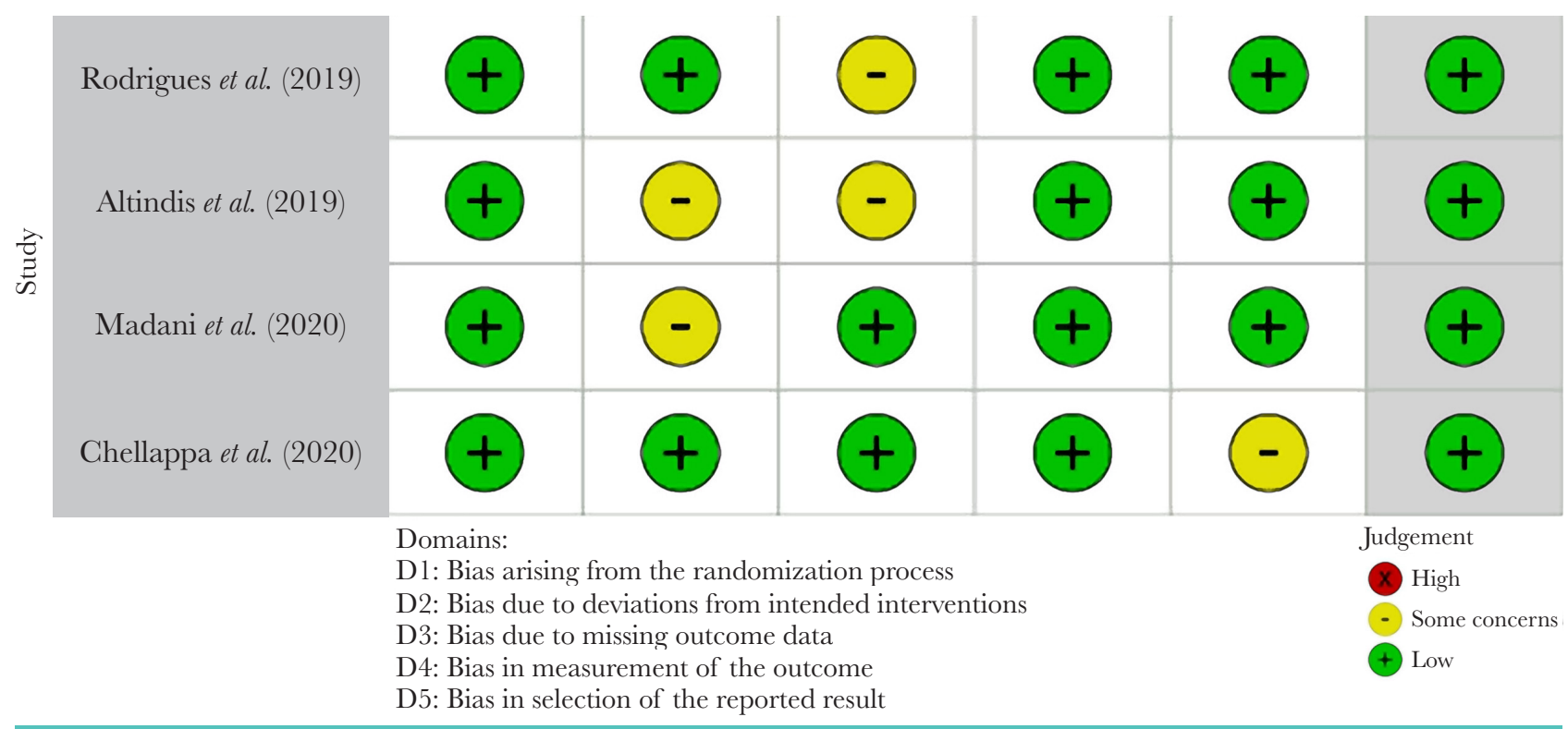

Figure 3. Continued.

array of therapeutic protocols with pain amelioration as the primary outcome [41]. Pain reduction also results in improved jaw motion, chewing, and masticatory efficiency [4]. The results in this systematic review were in coherence with the published literature, as most of the included studies in our review considered pain amelioration as the primary outcome of laser therapy.

Restriction or deflection in the range of mandibular movements (MO, LE and PE mandibular movements) and joint clicking are other frequent manifestations of TMDs. TMD patients also frequently report loss of masticatory efficacy. The masticatory patterns should be evaluated, and a definitive therapeutic protocol should be planned. Surface EMG, myofunctional procedure ratings, and assessment of masticatory efficiency are some of the employed objective approaches [42]. This systematic review also focused on improving the secondary outcomes like MO [5, 9, 10, 14-16, 26, 28, 30, 34, 37, 43-47], LE and PE mandibular movements [10, 14-16, 26, 28, 34, 37, 43-46, 48], PPT [14, 34, 35, 44, 46, 49, 50], EMG parameters [32, 34], joint noises [5, 28], TMD masticatory efficiency (ME) [49, 51], subjective tinnitus [29], and occlusal contacts distribution [48].

The importance of psychological factors (stress, anxiety, depression, and personality changes) has been thoroughly investigated in the etiopathogenesis of TMDs over the years. Published literature has demonstrated that the interrelation between stress, anxiety, depression, and distinct physical manifestations of TMDs is universally in sync with manifestations that are similar to those seen in other chronic musculoskeletal pain disorders [52]. Approximately 75\% of TMD patients exhibit chronic features, with detrimental biopsychosocial outcomes like depression and somatization [12]. In our systematic review, two studies emphasized the role of LLLT in improving TMD-related psychological and emotional aspects [24, 27]. The World Association of Laser Therapy came to a consensus in 2004 on the design of clinical trials with LLLT in TMDs. According to the established protocol, the placebo group should compulsorily be a part of the study design [53]. Most of the included RCTs involved a comparison of LLLT and placebo groups. However, 7 RCTs involved a comparison of laser with other interventions or compared co-interventions equally to both LLLT and placebo groups (Table 1).

Therapeutic lasers are generally close to the electromagnetic radiation spectrum and vary from visible (red) to invisible (infrared) light. The most used wavelengths usually range between 600 and $1000 \mathrm{~nm}$, permitting deeper penetration, relatively poor absorption, and easier transmission through the skin and mucous membranes [30].

In this systematic review, most of the studies used lasers with wavelengths within the electromagnetic radiation spectrum. The wavelengths ranged between $632.8 \mathrm{~nm}$ and $1064 \mathrm{~nm}$. Only five studies used lasers with wavelengths in the red range (shorter than $780 \mathrm{~nm}$ ). RCTs conducted by Altindis et al. [25] and Rodriguez et al. [27] did not mention the wavelength of the used lasers. Published literature has ascertained that combining lasers of two wavelengths have furnished positive outcomes. Lasers exert distinct effects in various biological tissues, explaining the variable results of laser therapy with different wavelengths [30]. In our systematic review, a combination of two laser types at different wavelengths was demonstrated by Shirani et al. [28], who used InGaAlP (660 nm) and GaAs (890 nm) lasers, Demirkol et al. [29], who used Nd: YAG (1064 nm) and diode laser (810 nm), and Pereira et al. [30], who used red laser (660 nm) and infrared laser $(795 \mathrm{~nm})$.

LLLT may show heterogeneity in the dose, power, and application time, with an irradiance of $5 \mathrm{~mW} / \mathrm{cm} 2$ to $5 \mathrm{~W} / \mathrm{cm} 2$, power range between $1 \mathrm{~mW}$ up to $10 \mathrm{~W}$, with pulsed or continuous beams, and the application span of 30-60 s/point [54]. The measure of the laser 


\section{JOURNAL of MEDICINE and LIFE}

Table 1. Characteristics of the included studies.

\begin{tabular}{|c|c|c|c|c|c|c|}
\hline Author & Sample size (n) & Age/gender & $\begin{array}{l}\text { Treatment } \\
\text { design }\end{array}$ & $\begin{array}{l}\text { Type of laser, } \\
\text { dose }\left(\mathrm{j} / \mathrm{cm}^{2}\right) \text { and } \\
\text { power }(\mathrm{mw}) \text { of } \\
\text { laser used }\end{array}$ & Outcome measures & Results \\
\hline $\begin{array}{l}\text { Shobha } \\
\text { et al. [5] } \\
(2017)\end{array}$ & $\begin{array}{l}\mathrm{n}=40 \\
\text { Group } 1 \text { (Laser } \\
\text { group } \mathrm{n}=20) \\
\text { Group } 2 \text { (placebo } \\
\text { group } \mathrm{n}=20 \text { ) }\end{array}$ & $\begin{array}{l}18-40 \text { yrs } \\
\text { Not mentioned }\end{array}$ & $\begin{array}{c}\text { Laser (20) } \\
\text { versus } \\
\text { placebo (20) }\end{array}$ & $\begin{array}{l}\text { Diode laser } \\
\text { (gallium } \\
\text { aluminum } \\
\text { arsenide, } 810 \mathrm{~nm} \text {, } \\
0.1 \mathrm{~W}, 6 \mathrm{~J} / \mathrm{cm}^{2} \text { ). }\end{array}$ & $\begin{array}{l}\mathrm{PI} \text { at function and at } \\
\text { rest (VAS), MO and } \\
\text { temporomandibular } \\
\text { clicking }\end{array}$ & $\begin{array}{c}\text { - } \downarrow \text { pain observed in both } \\
\text { active LLLT and placebo } \\
\text { groups } \\
\text { - improvement in clicking }\end{array}$ \\
\hline $\begin{array}{l}\text { Carli } \\
\text { et al. [9] } \\
(2016)\end{array}$ & $\begin{array}{c}\mathrm{n}=15 \\
\text { Group } 1 \text { (Laser } \\
\text { group } \mathrm{n}=8 \text { ) } \\
\text { Group } 2 \\
\text { (Botulinum toxin } \\
\text { A } \mathrm{n}=7 \text { ) }\end{array}$ & $\begin{array}{c}\text { Mean } \\
\text { age=28 yrs } \\
\text { M: F=2:13 }\end{array}$ & $\begin{array}{l}\text { Laser (8) } \\
\text { versus } \\
\text { Botulinum } \\
\text { toxin A (7) }\end{array}$ & $\begin{array}{l}\text { GaAlAs } 890 \mathrm{~nm} \text {, } \\
100 \mathrm{~mW}, 80 \mathrm{~J} / \mathrm{cm}^{2}\end{array}$ & PI (VAS) and MO & $\begin{array}{l}\text { Both Laser and Botulinum } \\
\text { toxin A treatments were } \\
\text { efficient in reducing } \\
\text { pain, but laser therapy } \\
\text { was much faster } \\
\text { in pain diminution. } \\
\text { (LLLT>Botulinum toxin A in } \\
\text { pain resolution). However, } \\
\text { both treatments showed } \\
\text { no statistically significant } \\
\text { improvement in MO. }\end{array}$ \\
\hline $\begin{array}{l}\text { Ahrari } \\
\text { et al. [10] } \\
(2014)\end{array}$ & $\begin{array}{l}\mathrm{n}=20 \\
\text { Group } 1 \text { (laser } \\
\text { group } \mathrm{n}=10 \text { ) } \\
\text { Group } 2 \text { (placebo } \\
\text { group } \mathrm{n}=10 \text { ) }\end{array}$ & $\begin{array}{l}\text { Mean age } \\
35.5 \text { yrs, } \\
20 \text { Females }\end{array}$ & $\begin{array}{l}\text { Laser (10) } \\
\text { versus } \\
\text { placebo (10) }\end{array}$ & $\begin{array}{l}\text { GaAIAs } 810 \mathrm{~nm} \text {, } \\
50 \mathrm{~mW}, 3.4 \mathrm{~J} / \mathrm{cm}^{2}\end{array}$ & $\begin{array}{l}\text { PI, mandibular } \\
\text { movements }\end{array}$ & LLLT>placebo \\
\hline $\begin{array}{l}\text { Chellappa } \\
\text { et al. [11] } \\
(2020)\end{array}$ & $\begin{array}{l}\mathrm{n}=60 \\
\text { Group } 1(\text { LLLT } \\
\text { group } \mathrm{n}=30) \\
\text { Group } 2 \text { (TENS } \\
\text { group } \mathrm{n}=30)\end{array}$ & Not mentioned & $\begin{array}{l}\text { LLLT group }(30) \\
\text { TENS group } \\
(30) \mathrm{n}=60\end{array}$ & $\begin{array}{l}672 \mathrm{~nm} \text { diode } \\
\text { laser } 50 \mathrm{~mW} \text {, } \\
3 \mathrm{~J} / \mathrm{cm}^{2}\end{array}$ & $\begin{array}{c}\mathrm{PI} \text { and range of } \\
\text { mandibular motion }\end{array}$ & LLLT>TENS \\
\hline $\begin{array}{l}\text { Ferreira } \\
\text { et al. [12] } \\
(2013)\end{array}$ & $\begin{array}{l}\mathrm{n}=40 \\
\text { Group } 1 \text { (laser } \\
\text { group } \mathrm{n}=20) \\
\text { Group } 2 \text { (placebo } \\
\text { group } \mathrm{n}=20 \text { ) }\end{array}$ & $\begin{array}{l}20-40 \text { yrs } \\
40 \text { females }\end{array}$ & $\begin{array}{c}\text { Laser (20) } \\
\text { versus } \\
\text { placebo (20) }\end{array}$ & $\begin{array}{c}\text { GaAIAs } 780 \mathrm{~nm}, \\
112.5 \mathrm{~J} / \mathrm{cm}^{2}, \\
50 \mathrm{~mW}\end{array}$ & $\mathrm{PI}$ & LLLT>placebo \\
\hline $\begin{array}{l}\text { Emshoff } \\
\text { et al. [13] } \\
(2008)\end{array}$ & $\begin{array}{c}\mathrm{n}=52 \\
\text { Group } 1 \text { (Study } \\
\text { group } \mathrm{n}=26 \text { ) } \\
\text { Group } 2 \\
\text { (control-placebo } \\
\mathrm{n}=26 \text { ) }\end{array}$ & $\begin{array}{l}18-58 \text { yrs } \\
M: F=10: 42\end{array}$ & $\begin{array}{c}\text { Laser (26) } \\
\text { versus } \\
\text { placebo (26) }\end{array}$ & $\begin{array}{c}\mathrm{HeNe} 632.8 \mathrm{~nm} \text {, } \\
1.5 \mathrm{~J} / \mathrm{cm}^{2} \text { and } \\
30 \mathrm{~mW}\end{array}$ & $\mathrm{PI}$ & LLLT=placebo \\
\hline $\begin{array}{l}\text { Venancio } \\
\text { et al. [14] } \\
(2005)\end{array}$ & $\begin{array}{c}\mathrm{n}=30 \\
\text { Group } 1 \text { (Study } \\
\text { group } \mathrm{n}=15 \text { ) } \\
\text { Group } 2 \\
\text { (control-placebo } \\
\mathrm{n}=15 \text { ) }\end{array}$ & $\begin{array}{l}\text { Not mentioned } \\
\text { M: } F=5: 25\end{array}$ & $\begin{array}{c}\text { Laser (15) } \\
\text { versus } \\
\text { placebo (15) }\end{array}$ & $\begin{array}{c}\text { GaAlAs } 780 \mathrm{~nm} \text {, } \\
6.3 \mathrm{~J} / \mathrm{cm}^{2} \text { and } \\
30 \mathrm{~mW}\end{array}$ & $\begin{array}{l}\text { PI, mandibular } \\
\text { function, pain } \\
\text { sensitivity }\end{array}$ & LLLT=placebo \\
\hline $\begin{array}{l}\text { Marini } \\
\text { et al. [15] } \\
(2010)\end{array}$ & $\begin{array}{c}\mathrm{n}=99 \\
\text { Group } 1 \\
\text { (Study/laser } \\
\text { group } \mathrm{n}=39 \text { ) } \\
\text { Group } 2 \\
\text { (ibuprofen } \mathrm{n}=30 \text { ) } \\
\text { Group } 3 \\
\text { (control-placebo } \\
\mathrm{n}=30 \text { ) }\end{array}$ & Not mentioned & $\begin{array}{c}\text { Laser (39) } \\
\text { versus } \\
\text { ibuprofen (30) } \\
\text { versus } \\
\text { placebo (30) }\end{array}$ & $\begin{array}{c}\text { GaAIAs } 910 \mathrm{~nm}, \\
400 \mathrm{~mW}\end{array}$ & $\begin{array}{l}\text { PI, mandibular } \\
\text { function, } \\
\text { morphologic } \\
\text { structural analysis } \\
\text { of TMJ }\end{array}$ & LLLT >placebo \\
\hline $\begin{array}{l}\text { Wang } \\
\text { et al. [16] } \\
(2011)\end{array}$ & $\begin{array}{c}\mathrm{n}=42 \\
\text { Group } 1 \text { (Study } \\
\text { group } \mathrm{n}=21 \text { ) } \\
\text { Group } 2 \\
\text { (control-placebo } \\
\mathrm{n}=21 \text { ) }\end{array}$ & Not mentioned & $\begin{array}{c}\text { Laser (21) } \\
\text { versus } \\
\text { placebo (21) }\end{array}$ & $\begin{array}{c}\text { GaAlAs } \\
650 \mathrm{~nm} / 830 \mathrm{~nm}, \\
300 \mathrm{~mW}\end{array}$ & $\begin{array}{c}\text { PI, functional } \\
\text { examination } \\
\text { (MO, lateral and } \\
\text { protrusive excursive } \\
\text { movements) }\end{array}$ & LLLT > placebo \\
\hline
\end{tabular}




\section{JOURNAL of MEDICINE and LIFE}

Table 1. Continued.

\begin{tabular}{|c|c|c|c|c|c|}
\hline $\begin{array}{l}\text { Brochado } \\
\text { et al. [24] } \\
\text { (2018) }\end{array}$ & $\begin{array}{c}\mathrm{n}=51 \\
\text { Group 1 (photo } \\
\text { biomodulation } \\
\text { (PBM) group } \\
\mathrm{n}=18 \text { ) } \\
\text { Group 2 (Manual } \\
\text { therapy group } \\
\mathrm{n}=16 \text { ) } \\
\text { Group } 3 \\
\text { (Combined group } \\
\mathrm{n}=17 \text { ) }\end{array}$ & $\begin{array}{l}21-77 \text { Yrs } \\
M: F=3: 48\end{array}$ & $\begin{array}{c}\text { PBM group (18) } \\
\text { Manual } \\
\text { therapy group } \\
\text { (16) } \\
\text { Combined } \\
\text { group (17) }\end{array}$ & $\begin{array}{c}\text { PBM with } 808 \mathrm{~nm} \text {, } \\
100 \mathrm{~mW}, \\
13.3 \mathrm{~J} / \mathrm{cm}^{2}\end{array}$ & $\begin{array}{l}\text { PI, mandibular } \\
\text { movements, } \\
\text { psychosocial } \\
\text { aspects, and anxiety } \\
\text { symptoms in TMD } \\
\text { patients }\end{array}$ \\
\hline
\end{tabular}

Altindis

et al. [25]

(2019)
$18-45$ yrs Not mentioned
Laser (10)

stabilization splint (10)
PI, muscle sensitivity and the superficial skin temperature differences
All protocols tested were able to promote pain relief, improve mandibular function, and reduce the negative psychosocial aspects and levels of anxiety in TMD patients. However, the combination of PBM and MT did not promote an increase in the effectiveness of both therapies alone.

Occlusal splint therapy and LLLT were effective in the treatment of MPS, and when thermographic data were considered, LLLT treatments could provide more advantageous results in these patients.

Both LLLT and LAT were effective in reducing pain and increasing excursive and protrusive mandibular motion in TMD patients. LAT could be suggested as a suitable alternative to LLLT, as it provided effective results while taking less chair time. range of motion (Lateral excursive and protrusive

\section{movements) $\mathrm{Pl}$ and Mouth opening \\ (15) \\ $21 \mathrm{~J} / \mathrm{cm}^{2}$}

LLLT improved the physical and emotional symptoms of TMD, with results like splint therapy.

\section{Rodrigues \\ et al. 27}

(2019)

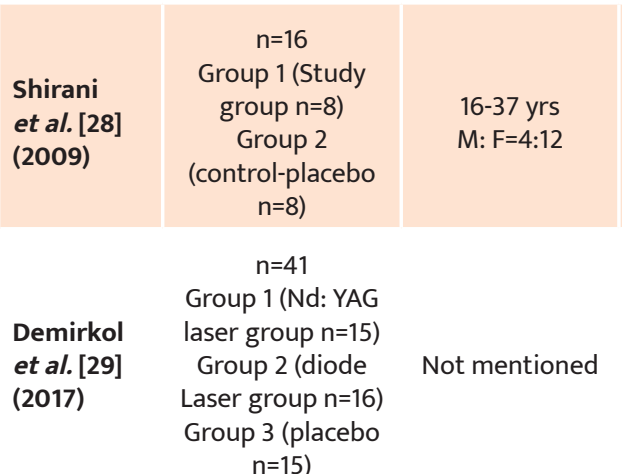

N/A

Laser (the
combination
of two
wavelengths, 8)
versus
placebo (8)

Nd: YAG laser

(15)

versus

diode laser (16) versus placebo

\begin{tabular}{|c|c|c|}
\hline $\begin{array}{l}\text { Pereira } \\
\text { et al. [30] } \\
(2014)\end{array}$ & $n=19$ & $\begin{array}{l}21-55 \text { yrs } \\
M: F=4: 15\end{array}$ \\
\hline $\begin{array}{l}\text { Demirkol } \\
\text { et al. [31] } \\
(2014)\end{array}$ & $\begin{array}{c}\mathrm{n}=30 \\
\text { Group } 1 \text { (laser } \\
\text { group } \mathrm{n}=10 \text { ) } \\
\text { Group } 2 \text { (occlusal } \\
\text { splint group } \mathrm{n}=10 \text { ) } \\
\text { Group } 3 \text { (placebo } \\
\mathrm{n}=10 \text { ) }\end{array}$ & Not mentioned \\
\hline
\end{tabular}

(15)

N/A

\begin{tabular}{|c|c|c|}
\hline N/A & $\begin{array}{c}660 \mathrm{~nm} \text { (red } \\
\text { laser) and } \\
795 \mathrm{~nm} \text { (infrared) } \\
\text { laser } \\
8 \mathrm{~J} / \mathrm{cm}^{2} \text { in Muscles } \\
4 \mathrm{~J} / \mathrm{cm}^{2} \text { in Joint }\end{array}$ & $\mathrm{PI}$ \\
\hline $\begin{array}{c}\text { Laser (10) } \\
\text { versus } \\
\text { occlusal splint } \\
(10) \\
\text { versus } \\
\text { placebo (10) }\end{array}$ & $\begin{array}{l}\text { Nd: YAG } 1064 \mathrm{~nm} \text {, } \\
250 \mathrm{~mW}, 8 \mathrm{~J} / \mathrm{cm}^{2}\end{array}$ & $\mathrm{PI}$ \\
\hline
\end{tabular}

N/A

InGaAIP 660 nm and GaAs $890 \mathrm{~nm}$,

$6.2 \mathrm{~J} / \mathrm{cm}^{2}$ and

$1.0 \mathrm{~J} / \mathrm{cm}^{2}$,

$17.3 \mathrm{~mW}$ and

$1.76 \mathrm{~mW}$
Nd: YAG laser

(1064 nm), diode

laser $(810 \mathrm{~nm})$,

$250 \mathrm{~mW}, 8 \mathrm{~J} / \mathrm{cm}^{2}$
The severity of the tinnitus (VAS)

LLLT >placebo

\section{LLLT >placebo}

Both lasers are effective in the treatment and remission of TMD symptoms 


\section{JOURNAL of MEDICINE and LIFE}

Table 1. Continued.

\begin{tabular}{|c|c|c|c|c|c|c|}
\hline $\begin{array}{l}\text { Venezian } \\
\text { et al. [32] } \\
(2010)\end{array}$ & $\begin{array}{c}n=48 \\
\text { Group } 1 \text { (Study } \\
\text { group } n=24 \text { ) } \\
\text { Group 2 } \\
\text { (control-placebo } \\
n=24 \text { ) }\end{array}$ & $\begin{array}{l}18-60 \text { yrs } \\
M: F=5: 43\end{array}$ & $\begin{array}{l}\text { Laser (24) } \\
\text { versus } \\
\text { placebo (24) }\end{array}$ & $\begin{array}{l}\text { GaAlAs } 780 \mathrm{~nm}, \\
25 \mathrm{~J} / \mathrm{cm}^{2} \text { or } \\
60 \mathrm{~J} / \mathrm{cm}^{2}, 50 \mathrm{~mW} \\
\text { or } 60 \mathrm{~mW}\end{array}$ & $\mathrm{PI}$ and EMG Activity & $\begin{array}{c}\text { LLLT> placebo }(\mathrm{PI}) \\
\text { LLLT= } \begin{array}{c}\text { placebo }(\mathrm{EMG} \\
\text { Activity) }\end{array}\end{array}$ \\
\hline $\begin{array}{l}\text { Cunha } \\
\text { et al. [33] } \\
\text { (2008) }\end{array}$ & $\begin{array}{c}\mathrm{n}=40 \\
\text { Group } 1 \text { (Study } \\
\text { group } \mathrm{n}=20 \text { ) } \\
\text { Group 2 } \\
\text { (control-placebo } \\
\mathrm{n}=20 \text { ) }\end{array}$ & $\begin{array}{c}20-68 \text { yrs } \\
\text { Not mentioned }\end{array}$ & $\begin{array}{l}\text { Laser (20) } \\
\text { versus } \\
\text { placebo (20) }\end{array}$ & $\begin{array}{c}\text { GaAlAs } 830 \mathrm{~nm}, \\
100 \mathrm{~J} / \mathrm{cm}^{2} \text { and } \\
500 \mathrm{~mW}\end{array}$ & $\mathrm{PI}$ and TMD status & LLLT=placebo \\
\hline $\begin{array}{l}\text { Uemoto } \\
\text { et al. [34] } \\
\text { (2013) }\end{array}$ & $\begin{array}{c}n=21 \\
\text { Group } 1 \text { (laser } \\
\text { group } n=7) \\
\text { Group } 2 \text { (needling } \\
\text { group } n=7 \text { ) } \\
\text { Group } 3(\text { (placebo } \\
n=7 \text { ) }\end{array}$ & $\begin{array}{l}20-50 \text { yrs } \\
28 \text { females }\end{array}$ & $\begin{array}{c}\text { Laser (7) } \\
\text { versus } \\
\text { needling group } \\
\text { (7) } \\
\text { versus } \\
\text { placebo (7) }\end{array}$ & $\begin{array}{l}\text { Laser type N/A } \\
795 \mathrm{~nm}, 4 \mathrm{~J} / \mathrm{cm}^{2} \text { or } \\
8 \mathrm{~J} / \mathrm{cm}^{2}, 80 \mathrm{~mW}\end{array}$ & $\begin{array}{l}\text { PI, EMG activity, } \\
\text { pain sensitivity, } \\
\text { mandibular } \\
\text { movements }\end{array}$ & LLLT>placebo (only $4 \mathrm{~J} / \mathrm{cm}^{2}$ ) \\
\hline $\begin{array}{l}\text { Oz S } \\
\text { et al. [35] } \\
(2010)\end{array}$ & $\begin{array}{c}\mathrm{n}=40 \\
\text { Group } 1 \text { (Study } \\
\text { group } \mathrm{n}=20 \text { ) } \\
\text { Group } 2 \\
\text { (control-occlusal } \\
\text { splints } \mathrm{n}=20 \text { ) }\end{array}$ & $\begin{array}{c}\text { Mean age } \\
32.8 \text { yrs } \\
M: F=6: 34\end{array}$ & $\begin{array}{c}\text { Laser (20) } \\
\text { versus } \\
\text { occlusal splints } \\
\text { (20) }\end{array}$ & $\begin{array}{l}\text { Laser type N/A } \\
820 \mathrm{~nm}, 3 \mathrm{~J} / \mathrm{cm}^{2} \\
\text { and } 300 \mathrm{~mW}\end{array}$ & $\begin{array}{l}\mathrm{Pl} \text {, mandibular } \\
\text { movements and } \\
\text { pressure pain } \\
\text { threshold }\end{array}$ & LLLT $=$ occlusal splints \\
\hline $\begin{array}{l}\text { Cavalcanti } \\
\text { et al. [36] } \\
(2016)\end{array}$ & $\begin{array}{c}n=60 \\
\text { Group } 1 \text { (laser } \\
\text { group } n=20) \\
\text { Group } 2 \text { (PDP } \\
\text { group } n=20 \text { ) } \\
\text { Group } 3 \text { (placebo } \\
n=20 \text { ) }\end{array}$ & $\begin{array}{l}20-50 \text { Yrs } \\
60 \text { females }\end{array}$ & $\begin{array}{l}\text { Laser (20) } \\
\text { versus } \\
\text { PDP (20) } \\
\text { versus } \\
\text { placebo (20 }\end{array}$ & $\begin{array}{l}\text { GaAlAs } 780 \mathrm{~nm} \text {, } \\
30 \mathrm{~mW}, 35 \mathrm{~J} / \mathrm{cm}^{2}\end{array}$ & $\begin{array}{c}\text { Presence/absence } \\
\text { of Pain }\end{array}$ & LLLT>placebo \\
\hline $\begin{array}{l}\text { Carli } \\
\text { et al. [37] } \\
\text { (2012) }\end{array}$ & $\begin{array}{c}\mathrm{n}=32 \\
\text { Group } 1 \text { (Laser + } \\
\text { piroxicam group } \\
\mathrm{n}=11 \text { ) } \\
\text { Group } 2 \text { (laser + } \\
\text { placebo piroxicam } \\
\mathrm{n}=11 \text { ) } \\
\text { Group } 3 \text { (placebo } \\
\text { laser + piroxicam } \\
\mathrm{n}=10 \text { ) }\end{array}$ & $\begin{array}{l}18-58 \text { yrs } \\
M: F=3: 29\end{array}$ & $\begin{array}{c}\text { Laser + } \\
\text { piroxicam (11) } \\
\text { versus } \\
\text { laser + placebo } \\
\text { piroxicam (11) } \\
\text { versus } \\
\text { placebo laser + } \\
\text { piroxicam (10) }\end{array}$ & $\begin{array}{c}\text { GaAlAs } 830 \mathrm{~nm}, \\
100 \mathrm{~J} / \mathrm{cm}^{2} \text { and } \\
100 \mathrm{~mW}\end{array}$ & $\begin{array}{l}\text { PI, functional } \\
\text { examination } \\
\text { (MO, lateral and } \\
\text { protrusive excursive } \\
\text { movements) }\end{array}$ & LLLT=placebo \\
\hline $\begin{array}{l}\text { Fornaini } \\
\text { et al. [38] } \\
\text { (2015) }\end{array}$ & $\begin{array}{l}\quad \mathrm{n}=24 \\
\text { Group } 1 \text { (laser } \\
\text { group } \mathrm{n}=12 \text { ) } \\
\text { Group } 2 \text { (placebo } \\
\text { group } \mathrm{n}=12 \text { ) }\end{array}$ & $\begin{array}{l}17-64 \text { Yrs } \\
M: F=5: 19\end{array}$ & $\begin{array}{l}\text { Laser (10) } \\
\text { versus } \\
\text { placebo (10) }\end{array}$ & $\begin{array}{l}\text { GaAs } 904 \mathrm{~nm} \text {, } \\
15 \mathrm{~mW}, 6 \mathrm{~J} / \mathrm{cm}^{2}\end{array}$ & $\mathrm{PI}$ & LLLT>placebo \\
\hline $\begin{array}{l}\text { Mazzetto } \\
\text { et al. [43] } \\
(2010)\end{array}$ & $\begin{array}{c}\mathrm{n}=40 \\
\text { Group } 1 \text { (Study } \\
\text { group } \mathrm{n}=20 \text { ) } \\
\text { Group 2 } \\
\text { (control-placebo } \\
\mathrm{n}=20 \text { ) }\end{array}$ & Not mentioned & $\begin{array}{l}\text { Laser (20) } \\
\text { versus } \\
\text { placebo (20) }\end{array}$ & $\begin{array}{l}\text { GaAlAs } 830 \mathrm{~nm}, \\
5 \mathrm{~J} / \mathrm{cm}^{2} \text { and } \\
40 \mathrm{~mW}\end{array}$ & $\begin{array}{l}\mathrm{PI} \text {, mandibular } \\
\text { movements }\end{array}$ & LLLT>placebo \\
\hline $\begin{array}{l}\text { Röhlig } \\
\text { et al. [44] } \\
\text { (2011) }\end{array}$ & $\begin{array}{c}\mathrm{n}=40 \\
\text { Group } 1 \text { (laser } \\
\text { group } \mathrm{n}=20 \text { ) } \\
\text { Group 2 } \\
\text { (control-placebo } \\
\mathrm{n}=20 \text { ) }\end{array}$ & Not mentioned & $\begin{array}{l}\text { Laser (20) } \\
\text { versus } \\
\text { placebo (20) }\end{array}$ & $\begin{array}{l}\text { GaAs } 820 \mathrm{~nm}, \\
300 \mathrm{~mW}, 8 \mathrm{~J} / \mathrm{cm}^{2}\end{array}$ & $\begin{array}{l}\text { PI, functional } \\
\text { examination, pain } \\
\text { sensitivity }\end{array}$ & LLLT>placebo \\
\hline
\end{tabular}




\section{JOURNAL of MEDICINE and LIFE}

\begin{tabular}{|c|c|c|c|c|c|c|}
\hline $\begin{array}{l}\text { Silva } \\
\text { et al. [45] } \\
\text { (2012) }\end{array}$ & $\begin{array}{c}\mathrm{n}=45 \\
\text { Group } 1 \text { (low } \\
\text { energy level } \\
\text { group } \mathrm{n}=15 \text { ) } \\
\text { Group } 2 \text { (high } \\
\text { energy level } \\
\text { group } \mathrm{n}=15 \text { ) } \\
\text { Group } 3 \text { (placebo } \\
\mathrm{n}=15 \text { ) }\end{array}$ & $\begin{array}{l}25-53 \text { yrs } \\
M: F=15: 30\end{array}$ & $\begin{array}{l}\text { Low energy } \\
\text { laser (15) } \\
\text { versus } \\
\text { high energy } \\
\text { laser (15) } \\
\text { versus } \\
\text { placebo (15) }\end{array}$ & $\begin{array}{l}\text { GaAlAs } 780 \mathrm{~nm} \text {, } \\
52 \mathrm{~J} / \mathrm{cm}^{2} \text { and } \\
105 \mathrm{~J} / \mathrm{cm}^{2}, 70 \mathrm{~mW}\end{array}$ & $\begin{array}{l}\mathrm{PI}, \text { mandibular } \\
\text { movements }\end{array}$ & LLLT>placebo \\
\hline $\begin{array}{l}\text { Sancakli } \\
\text { et al. [46] } \\
\text { (2016) }\end{array}$ & $\begin{array}{c}\mathrm{n}=30 \\
\text { Group } 1 \text { (laser } \\
\text { group I } \mathrm{n}=10 \text { ) } \\
\text { Group } 2 \text { (laser } \\
\text { group II group } \\
\mathrm{n}=10 \text { ) } \\
\text { Group } 3 \text { (placebo } \\
\mathrm{n}=10 \text { ) }\end{array}$ & $\begin{array}{l}18-60 \text { yrs } \\
M: F=9: 21\end{array}$ & $\begin{array}{l}\text { Laser I (10) } \\
\text { versus } \\
\text { laser II (10) } \\
\text { versus } \\
\text { placebo (10) }\end{array}$ & $\begin{array}{l}\text { GaAs } 820 \mathrm{~nm} \\
30 \mathrm{~mW}, 3 \mathrm{~J} / \mathrm{cm}^{2}\end{array}$ & $\begin{array}{l}\text { PI, mandibular } \\
\text { mobility, pain } \\
\text { sensitivity }\end{array}$ & LLLT>placebo \\
\hline $\begin{array}{l}\text { Costa } \\
\text { et al. [47] } \\
(2017)\end{array}$ & $\begin{array}{l}\quad \mathrm{n}=60 \\
\text { Group } 1 \text { (photo } \\
\text { biomodulation } \\
\text { (PBM) group } \\
\mathrm{n}=30 \text { ) }\end{array}$ & $\begin{array}{l}18-76 \text { yrs } \\
M: F=6: 54\end{array}$ & $\begin{array}{l}\text { PBM } \\
\text { group (30) } \\
\text { versus } \\
\text { placebo } \\
\text { group (30) }\end{array}$ & $\begin{array}{l}\text { infrared laser } \\
(830 \mathrm{~nm}) \\
100 \mathrm{~mW} \\
100 \mathrm{~J} / \mathrm{cm}^{2}\end{array}$ & $\begin{array}{l}\text { Referred pain } \\
\text { elicited by palpation } \\
\text { and maximum } \\
\text { mouth opening }\end{array}$ & $\begin{array}{l}\text { PBMT }(830 \mathrm{~nm}) \text { reduces } \\
\text { pain in algic points, but } \\
\text { does not influence the } \\
\text { extent of mouth opening } \\
\text { in patients with myalgia }\end{array}$ \\
\hline $\begin{array}{l}\text { Godoy } \\
\text { et al. [48] } \\
\text { (2015) }\end{array}$ & N/A & $\begin{array}{c}14-23 \text { yrs } \\
\text { Not mentioned }\end{array}$ & $\begin{array}{l}\text { Laser } \\
\text { versus } \\
\text { Placebo }\end{array}$ & $\begin{array}{l}\text { Laser type N/A } \\
780 \mathrm{~nm}, 50 \mathrm{~mW} \\
33.5 \mathrm{~J} / \mathrm{cm}^{2}\end{array}$ & $\begin{array}{l}\mathrm{PI} \text {, mandibular } \\
\text { range of motion } \\
\text { and occlusal } \\
\text { contacts }\end{array}$ & $\begin{array}{l}\text { No statistically significant } \\
\text { differences were found } \\
\text { regarding pain, mandibular } \\
\text { range of motion, or the } \\
\text { distribution of occlusal } \\
\text { contacts after treatment } \\
\text { with low-level laser } \\
\text { therapy. }\end{array}$ \\
\hline $\begin{array}{l}\text { Maia } \\
\text { et al. [49] } \\
(2014)\end{array}$ & $\begin{array}{l}\quad \mathrm{n}=21 \\
\text { Group } 1 \text { (laser } \\
\text { group } \mathrm{n}=11) \\
\text { Group } 2 \text { (placebo } \\
\text { group } \mathrm{n}=10)\end{array}$ & $\begin{array}{c}\text { Mean age } \\
27.7 \pm 1.44 \text { yrs } \\
M: F=2: 19\end{array}$ & $\begin{array}{l}\text { Laser (10) } \\
\text { versus } \\
\text { placebo (9) }\end{array}$ & $\begin{array}{l}\text { GaAlAs } 808 \mathrm{~nm} \text {, } \\
100 \mathrm{~mW}, 70 \mathrm{~J} / \mathrm{cm}^{2}\end{array}$ & $\begin{array}{l}\mathrm{PI} \text {, masticatory } \\
\text { performance, pain } \\
\text { sensitivity }\end{array}$ & LLLT>placebo \\
\hline $\begin{array}{l}\text { Magri } \\
\text { et al. [50] } \\
\text { (2017) }\end{array}$ & $\begin{array}{c}\mathrm{n}=91 \\
\text { Group } 1 \text { (laser } \\
\text { group } \mathrm{n}=31 \text { ) } \\
\text { Group } 2 \text { (placebo } \\
\text { group } \mathrm{n}=30 \text { ) } \\
\text { Group 3 (control } \\
\mathrm{n}=30 \text { ) }\end{array}$ & $\begin{array}{l}18-60 \text { Yrs } \\
91 \text { females }\end{array}$ & $\begin{array}{l}\text { Laser (31) } \\
\text { versus } \\
\text { placebo (30) } \\
\text { versus } \\
\text { control (30) }\end{array}$ & $\begin{array}{l}\text { GaAlAs } 780 \mathrm{~nm} \text {, } \\
\text { TMJ, } 20 \mathrm{~mW} \text {, } \\
\text { muscle, } 30 \mathrm{~mW} \text {, } \\
5 \text { or } 7.5 \mathrm{~J} / \mathrm{cm}^{2}\end{array}$ & $\begin{array}{l}\text { PI, pain sensitivity, } \\
\text { the sensory } \\
\text { and affective } \\
\text { dimensions of pain }\end{array}$ & LLLT=placebo \\
\hline $\begin{array}{l}\text { Carrasco } \\
\text { et al. [51] } \\
(2008)\end{array}$ & $\begin{array}{c}\mathrm{n}=14 \\
\text { Group } 1 \text { (Study } \\
\text { group } \mathrm{n}=7 \text { ) } \\
\text { Group 2 } \\
\text { (control-placebo } \\
\mathrm{n}=7 \text { ) }\end{array}$ & Not mentioned & $\begin{array}{l}\text { Laser (7) } \\
\text { versus } \\
\text { placebo (7) }\end{array}$ & $\begin{array}{l}\text { GaAlAs } 780 \mathrm{~nm} \\
105 \mathrm{~J} / \mathrm{cm}^{2} \text { and } \\
70 \mathrm{~mW}\end{array}$ & $\mathrm{PI}$ and $\mathrm{ME}$ & $\begin{array}{c}\text { LLLT>placebo (PI on } \\
\text { palpation) LLLT=placebo } \\
(\mathrm{ME})\end{array}$ \\
\hline $\begin{array}{l}\text { Frare } \\
\text { et al. [56] } \\
(2008)\end{array}$ & $\begin{array}{c}\mathrm{n}=18 \\
\text { Group } 1 \text { (Study } \\
\text { group } \mathrm{n}=10 \text { ) } \\
\text { Group 2 } \\
\text { (control-placebo } \\
\mathrm{n}=8 \text { ) }\end{array}$ & $\begin{array}{c}18-45 \text { yrs } \\
18 \text { females }\end{array}$ & $\begin{array}{l}\text { Laser (10) } \\
\text { versus } \\
\text { placebo (8) }\end{array}$ & $\begin{array}{l}\text { GaAs } 904 \mathrm{~nm} \\
70 \mathrm{~mW}, 6 \mathrm{~J} / \mathrm{cm}^{2}\end{array}$ & PI & LLLT>placebo \\
\hline $\begin{array}{l}\text { Mazzetto } \\
\text { et al. [57] } \\
(2007)\end{array}$ & $\begin{array}{c}\mathrm{n}=48 \\
\text { Group } 1 \text { (Study } \\
\text { group } \mathrm{n}=24 \text { ) } \\
\text { Group 2 } \\
\text { (control-placebo } \\
\mathrm{n}=24 \text { ) }\end{array}$ & Not mentioned & $\begin{array}{l}\text { Laser (24) } \\
\text { versus } \\
\text { placebo (24) }\end{array}$ & $\begin{array}{l}\text { GaAIAs } 780 \mathrm{~nm} \\
89.7 \mathrm{~J} / \mathrm{cm}^{2} \\
\text { and } 70 \mathrm{~mW}\end{array}$ & $\mathrm{PI}$ & LLLT>placebo \\
\hline
\end{tabular}




\section{JOURNAL of MEDICINE and LIFE}

Table 1. Continued.

\begin{tabular}{|c|c|c|c|c|c|c|}
\hline $\begin{array}{l}\text { Kulekcioglu } \\
\text { et al. [58] } \\
(2003)\end{array}$ & $\begin{array}{c}\mathrm{n}=35 \\
\text { Group } 1 \text { (Study } \\
\text { group } \mathrm{n}=20 \text { ) } \\
\text { Group } 2 \\
\text { (control-placebo } \\
\mathrm{n}=15 \text { ) }\end{array}$ & $\begin{array}{l}20-59 \text { yrs } \\
M: F=7: 28\end{array}$ & $\begin{array}{l}\text { Laser (20) } \\
\text { versus } \\
\text { placebo (15) }\end{array}$ & $\begin{array}{c}\text { GaAs } 904 \mathrm{~nm} \\
3 \mathrm{~J} / \mathrm{cm}^{2} \text { and } \\
17 \mathrm{~mW}\end{array}$ & $\begin{array}{l}\text { PI, mandibular } \\
\text { function (Mouth } \\
\text { opening: MO and } \\
\text { LM), TMJ sounds }\end{array}$ & $\begin{array}{c}\text { LLLT> placebo }(\mathrm{MO}, \mathrm{LM}) \\
\mathrm{LLLT}=\text { placebo }(\mathrm{PI}, \mathrm{TMJ} \\
\text { sounds })\end{array}$ \\
\hline $\begin{array}{l}\text { Machado } \\
\text { et al. [59] } \\
(2016)\end{array}$ & $\mathrm{n}=82$ & Not mentioned & $\begin{array}{l}\text { GI: laser + Oral } \\
\text { motor (OM) } \\
\text { exercises (21) } \\
\text { versus } \\
\text { GII: pain relief } \\
\text { strategies + OM } \\
\text { exercises (22) } \\
\text { versus } \\
\text { GIII laser } \\
\text { placebo + OM } \\
\text { exercises (21) } \\
\text { versus } \\
\text { GIV: laser (18) }\end{array}$ & $\begin{array}{c}\text { GaAlAs } 780 \mathrm{~nm}, \\
60 \mathrm{~mW} \\
60 \pm 1.0 \mathrm{~J} / \mathrm{cm}^{2}\end{array}$ & $\begin{array}{l}\text { PI, TMD severity, } \\
\text { and orofacial } \\
\text { myofunctional } \\
\text { status }\end{array}$ & LLLT=placebo \\
\hline
\end{tabular}

F - Female; GaAlAs - Gallium-aluminum-arsenide laser; GaAS - Gallium-arsenide laser; HeNe - Helium-neon laser; LAT - Laser acupuncture therapy; LLLT - Low-level laser therapy; LM - Lateral movements; ND: YAG - Neodymium-doped yttrium aluminum garnet; M - Male; ME - masticatory efficiency; MPS - Myofascial pain syndrome; MO - mouth opening; MT - Manual therapy; N/A: Not Applicable; OM - Oral motor; PBM - Photobiomodulation; PI - Pain intensity; TENS - Transcutaneous electrical nerve stimulation; TMD - temporomandibular joint dysfunction; VAS - visual analog scale.

Table 2. Details of the eligible studies.

\begin{tabular}{|l||c|c|c|}
\hline Author & $\begin{array}{c}\text { Treatment } \\
\text { time/number of total } \\
\text { of study }\end{array}$ & $\begin{array}{c}\text { Site of laser } \\
\text { application }\end{array}$ \\
\hline
\end{tabular}

\begin{tabular}{|c|c|c|c|c|c|}
\hline $\begin{array}{l}\text { Shobha et al. [5] } \\
\text { (2017) }\end{array}$ & India & $\begin{array}{l}\text { Indian Journal of } \\
\text { Dental research }\end{array}$ & $60 \mathrm{~s} / 8 / 2-3$ per week & TMJ and muscles & Follow-up after 30 days \\
\hline Carli et al. [9] (2016) & Brazil & $\begin{array}{c}\text { Journal of } \\
\text { Photochemistry and } \\
\text { Photobiology, } \\
\text { B: Biology }\end{array}$ & $\begin{array}{c}-/ 7 / 48 \text { hours interval } \\
\text { between each } \\
\text { session }\end{array}$ & Muscles & N/A \\
\hline
\end{tabular}

\begin{tabular}{|c|c|c|c|c|c|}
\hline $\begin{array}{l}\text { Ahrari et al. [10] } \\
(2014)\end{array}$ & Iran & $\begin{array}{l}\text { Lasers in Medical } \\
\text { Science }\end{array}$ & $120 \mathrm{~s} / 12 / 3$ & Muscles & $\begin{array}{l}\text { Before intervention, after six } \\
\text { applications, at the end of } \\
\text { treatment, and } 1 \text { month after } \\
\text { the last application }\end{array}$ \\
\hline $\begin{array}{l}\text { Chellappa et al. [11] } \\
(2020)\end{array}$ & India & $\begin{array}{l}\text { Indian Journal of } \\
\text { Dental research }\end{array}$ & $\begin{array}{c}120 \mathrm{~s} / 12 / \text { two } \\
\text { sessions/week for } 6 \\
\text { weeks }\end{array}$ & TMJ and muscles & N/A \\
\hline $\begin{array}{l}\text { Ferreira et al. [12] } \\
(2013)\end{array}$ & Brazil & $\begin{array}{l}\text { Lasers in Medical } \\
\text { Science }\end{array}$ & $90 \mathrm{~s} / 12 / 1$ & TMJ and Muscles & $\begin{array}{l}\text { Before intervention, monthly } \\
\text { until intervention completed }\end{array}$ \\
\hline $\begin{array}{l}\text { Emshoff et al. [13] } \\
(2008)\end{array}$ & Austria & $\begin{array}{l}\text { Oral Surgery, Oral } \\
\text { Medicine, Oral } \\
\text { Pathology, Oral } \\
\text { Radiology, and } \\
\text { Endodontics }\end{array}$ & $120 \mathrm{~s} / 20 / 2-3$ & TMJ & $\begin{array}{c}\text { Before treatment and } 2,4 \text {, and } \\
8 \text { weeks after the first laser } \\
\text { therapy }\end{array}$ \\
\hline
\end{tabular}

\begin{tabular}{|c|c|c|c|c|c|}
\hline $\begin{array}{l}\text { Venancio et al. [14] } \\
(2005)\end{array}$ & Brazil & $\begin{array}{l}\text { Journal of Oral } \\
\text { Rehabilitation }\end{array}$ & $10 \mathrm{~s} / 6 / 2$ & TMJ & $\begin{array}{l}\text { sessions, and at the follow-up } \\
\text { appointments after } 15,30, \\
\text { and } 60 \text { days of the end of }\end{array}$ \\
\hline
\end{tabular}




\section{JOURNAL of MEDICINE and LIFE}

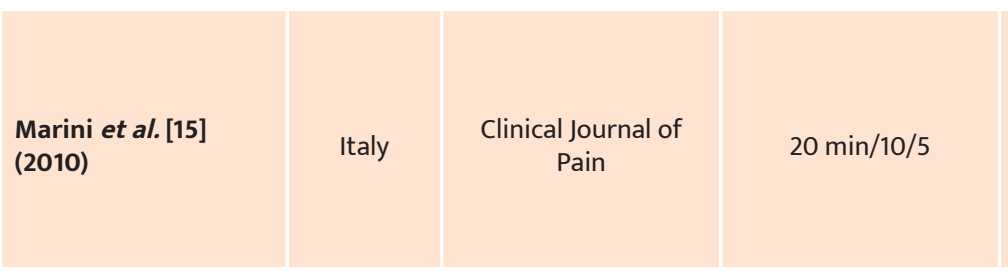

Wang et al. [16] (2011) China West China Journa

Brochado et al. [24]
(2018)

$15 \mathrm{~min} / 6 / 6$

TMJ

TMJ

PI at baseline, 2, 5, 10, and 15 days after treatment.

Mandibular function at baseline, 15 days and 1 month after treatment. MRI at baseline and at the end of the treatment.

Before treatment, immediately,

1 month and 2 months after treatment

(2018)

\section{Brazil Brazilian Oral}

Research

$40 \mathrm{~s}$ (joint); 21min

(muscle) $/ 12 / 3$

times a week for 4 consecutive weeks

TMJ and muscles

Follow-up after 4 and 8 weeks

Muscles

N/A

Altindis et al. [25]

Brazil

Complementary

(2019)

Medicine

Madani A et al. [26]

Iran

Lasers in Medical Science

$30 \mathrm{~s} / 10 /$ two times a

joint, muscles, and

week for 5 weeks

acupuncture points

Evaluated before treatment/after 5 sitting/10 sitting and 30 days after therapy

\begin{tabular}{|c|c|c|c|c|c|}
\hline $\begin{array}{l}\text { Rodrigues et al. } 27 \\
\text { (2019) }\end{array}$ & Brazil & $\begin{array}{l}\text { Complimentary } \\
\text { Therapies in } \\
\text { Medicine }\end{array}$ & N/A & TMJ and muscles & N/A \\
\hline $\begin{array}{l}\text { Shirani et al. [28] } \\
\text { (2009) }\end{array}$ & Iran & $\begin{array}{l}\text { Lasers in Medical } \\
\text { Science }\end{array}$ & $360 \mathrm{~s} / 6 / 2$ & Muscles & $\begin{array}{l}\text { Before and immediately } \\
\text { after treatment, } 1 \text { week after } \\
\text { treatment, and on the day of } \\
\text { feeling complete pain relief }\end{array}$ \\
\hline $\begin{array}{l}\text { Demirkol et al. [29] } \\
\text { (2017) }\end{array}$ & Turkey & $\begin{array}{l}\text { Photomedicine and } \\
\text { Laser Surgery }\end{array}$ & $20 \mathrm{~s}$ or $9 \mathrm{~s} / 10 / 5$ & $\begin{array}{l}\text { External Auditory } \\
\text { Meatus }\end{array}$ & $\begin{array}{l}\text { Before treatment, immediately } \\
\text { and } 1 \text { month after treatment }\end{array}$ \\
\hline $\begin{array}{l}\text { Pereira et al. [30] } \\
\text { (2014) }\end{array}$ & Brazil & $\begin{array}{l}\text { Cranio: The Journal } \\
\text { of Craniomandibular } \\
\text { and Sleep Practice }\end{array}$ & N/A & TMJ and Muscles & $\begin{array}{c}\text { Reassessed at } 24 \text { hours and } 30 \\
\text { days (short-term assessment), } \\
90 \text { days (medium-term), and } \\
180 \text { days (long-term) }\end{array}$ \\
\hline $\begin{array}{l}\text { Demirkol et al. [31] } \\
\text { (2014) }\end{array}$ & Turkey & $\begin{array}{l}\text { Lasers in Medical } \\
\text { Science }\end{array}$ & $20 \mathrm{~s} / 10 / 5$ & Muscles & $\begin{array}{c}\text { Before treatment, immediately } \\
\text { and } 3 \text { weeks after treatment }\end{array}$ \\
\hline $\begin{array}{l}\text { Venezian et al. [32] } \\
\text { (2010) }\end{array}$ & & $\begin{array}{l}\text { Cranio: The Journal } \\
\text { of Craniomandibular } \\
\text { and Sleep Practice }\end{array}$ & 20 or $40 \mathrm{~s} / 8 / 2$ & Muscles & $\begin{array}{c}\text { PI: before treatment, } \\
\text { immediately and } 30 \text { days after } \\
\text { treatment } \\
\text { EMG: before and immediately } \\
\text { after treatment }\end{array}$ \\
\hline $\begin{array}{l}\text { Cunha et al. [33] } \\
(2008)\end{array}$ & Brazil & $\begin{array}{l}\text { International Dental } \\
\text { Journal }\end{array}$ & $20 \mathrm{~s} / 4 / 1$ & TMJ and/or muscles & $\begin{array}{c}\text { Before treatment and after the } \\
\text { last treatment }\end{array}$ \\
\hline $\begin{array}{l}\text { Uemoto et al. [34] } \\
\text { (2013) }\end{array}$ & Brazil & $\begin{array}{l}\text { Journal of Oral } \\
\text { Science }\end{array}$ & $-/ 4 /-$ & Muscles & $\begin{array}{l}\text { Before treatment, after four } \\
\text { sessions with intervals ranging } \\
\text { between } 48 \text { and } 72 \mathrm{~h}\end{array}$ \\
\hline Oz S et al. [35] (2010) & Turkey & $\begin{array}{c}\text { Journal of } \\
\text { Craniofacial Surgery }\end{array}$ & $\mathrm{N} / \mathrm{A}$ & $-/ 10 / 2$ times per week & N/A \\
\hline $\begin{array}{l}\text { Cavalcanti et al. [36] } \\
(2016)\end{array}$ & Brazil & $\begin{array}{l}\text { Photomedicine and } \\
\text { Laser Surgery }\end{array}$ & $20 \mathrm{~s} / 12 / 3$ & TMJ and Muscles & $\begin{array}{c}\text { Before treatment, at each } \\
\text { week till the fourth week after } \\
\text { treatment }\end{array}$ \\
\hline Carli et al. [37] (2012) & Brazil & $\begin{array}{l}\text { Journal of Oral } \\
\text { Rehabilitation }\end{array}$ & $28 \mathrm{~s} / 4 / 2$ & TMJ and Muscles & $\begin{array}{l}\text { Before treatment, after the } \\
\text { first, second, third, and fourth } \\
\text { treatment sessions, and } 30 \\
\text { days after last treatment. }\end{array}$ \\
\hline
\end{tabular}




\section{JOURNAL of MEDICINE and LIFE}

\begin{tabular}{|c|c|c|c|c|c|}
\hline $\begin{array}{l}\text { Fornaini et al. [38] } \\
(2015)\end{array}$ & Italy & Laser Therapy & $15 \mathrm{~min} / 14 / 7$ & TMJ & $\begin{array}{l}\text { Before treatment, } 1 \text { and } 2 \\
\text { weeks after treatment }\end{array}$ \\
\hline $\begin{array}{l}\text { Mazzetto et al. [43] } \\
(2010)\end{array}$ & Brazil & $\begin{array}{l}\text { Brazilian Dental } \\
\text { Journal }\end{array}$ & $10 \mathrm{~s} / 8 / 2$ & TMJ & $\begin{array}{c}\text { Before treatment, immediately, } \\
7 \text { and } 30 \text { days after } \\
\text { applications }\end{array}$ \\
\hline $\begin{array}{l}\text { Röhlig et al. [44] } \\
\text { (2011) }\end{array}$ & Turkey & $\begin{array}{l}\text { Turkish Journal of } \\
\text { Physical Medicine } \\
\text { and Rehabilitation }\end{array}$ & $10 s / 10 / 3-4$ & Muscles & $\begin{array}{l}\text { Before treatment and after the } \\
\text { last applications }\end{array}$ \\
\hline Silva et al. [45] (2012) & Brazil & $\begin{array}{l}\text { Cranio: The Journal } \\
\text { of Craniomandibular } \\
\text { and Sleep Practice }\end{array}$ & $30 \mathrm{~s}$ or $60 \mathrm{~s} / 10 / 2$ & TMJ and/or Muscles & $\begin{array}{l}\text { Before treatment, immediately } \\
\text { after the first, fifth, tenth } \\
\text { treatments, and } 5 \text { weeks after } \\
\text { completing the applications }\end{array}$ \\
\hline $\begin{array}{l}\text { Sancakli et al. [46] } \\
(2016)\end{array}$ & Turkey & BMC Oral Health & $10 \mathrm{~s} / 12 / 3$ & Muscles & $\begin{array}{l}\text { Before treatment and after the } \\
\text { completion of therapy }\end{array}$ \\
\hline $\begin{array}{l}\text { Costa et al. [47] } \\
(2017)\end{array}$ & Brazil & $\begin{array}{l}\text { Brazilian Oral } \\
\text { Research }\end{array}$ & $28 \mathrm{~s} /-/-$ & Muscles & $\begin{array}{l}\text { Long-term evaluation (6 } \\
\text { months) }\end{array}$ \\
\hline $\begin{array}{l}\text { Godoy et al. [48] } \\
(2015)\end{array}$ & Brazil & $\begin{array}{l}\text { Journal of Oral and } \\
\text { Maxillofacial Surgery }\end{array}$ & $20 \mathrm{~s} /-/-$ & Muscles & N/A \\
\hline Maia et al. [49] (2014) & Brazil & $\begin{array}{l}\text { Lasers in Medical } \\
\text { Science }\end{array}$ & $19 \mathrm{~s} / 8 / 2$ & Muscles & $\begin{array}{l}\text { MP and PPT, before treatment, } \\
\text { at the end of treatment and } 30 \\
\text { days after treatment VAS, at } \\
\text { the same time as above; it was } \\
\text { also measured weekly }\end{array}$ \\
\hline $\begin{array}{l}\text { Magri et al. [50] } \\
(2017)\end{array}$ & Brazil & $\begin{array}{l}\text { Lasers in Medical } \\
\text { Science }\end{array}$ & $10 \mathrm{~s} / 8 / 2$ & TMJ and muscles & $\begin{array}{l}\text { Before treatment, after each } \\
\text { treatment and } 30 \text { days after } \\
\text { last treatment }\end{array}$ \\
\hline $\begin{array}{l}\text { Carrasco et al. [51] } \\
(2008)\end{array}$ & Brazil & $\begin{array}{l}\text { Cranio: The Journal } \\
\text { of Craniomandibular } \\
\text { and Sleep Practice }\end{array}$ & $60 \mathrm{~s} / 8 / 2$ & TMJ & $\begin{array}{l}\text { Before treatment, after the } 8^{\text {th }} \\
\text { application, } 30 \text { days after the } \\
\text { last application }\end{array}$ \\
\hline $\begin{array}{l}\text { Frare et al. [56] } \\
(2008)\end{array}$ & Brazil & $\begin{array}{l}\text { Revista Brasileira de } \\
\text { Fisioterapia }\end{array}$ & $16 \mathrm{~s} / 8 / 2$ & $\begin{array}{l}\text { TMJ and external } \\
\text { auditory meatus }\end{array}$ & $\begin{array}{l}\text { Before and immediately } \\
\text { after all sessions of laser } \\
\text { applications }\end{array}$ \\
\hline $\begin{array}{l}\text { Mazzetto et al. [57] } \\
(2007)\end{array}$ & Brazil & $\begin{array}{l}\text { Cranio: The Journal } \\
\text { of Craniomandibular } \\
\text { and Sleep Practice }\end{array}$ & $10 \mathrm{~s} / 8 / 2$ & $\begin{array}{c}\text { TMJ (external } \\
\text { auditory meatus) }\end{array}$ & $\begin{array}{l}\text { Before treatment, after the } \\
4^{\text {th }} \text { and } 8^{\text {th }} \text { applications, and } 30 \\
\text { days after the last application. }\end{array}$ \\
\hline $\begin{array}{l}\text { Kulekcioglu et al. [58] } \\
(2003)\end{array}$ & Turkey & $\begin{array}{l}\text { Scandinavian Journal } \\
\text { of Rheumatology }\end{array}$ & $180 \mathrm{~s} / 15 /-$ & TMJ and/or muscles & $\begin{array}{c}\text { Before, after, and } 1 \text { month after } \\
\text { treatment }\end{array}$ \\
\hline $\begin{array}{l}\text { Machado et al. [59] } \\
\text { (2016) }\end{array}$ & Brazil & $\begin{array}{l}\text { Lasers in Medical } \\
\text { Science }\end{array}$ & $45 \mathrm{~min} / 12 / 1-0.5$ & TMJ and Muscles & $\begin{array}{l}\text { Before treatment, immediately } \\
\text { and } 1 \text { month after treatment }\end{array}$ \\
\hline
\end{tabular}

EMG - electromyography; MRI - magnetic resonance imaging; PI - Pain intensity; PPT - Pressure pain threshold; TMJ - temporomandibular joint; VAS - visual analog scale.

effect is also determined by the laser dose. According to Bjordal et al. [55], the debate on the efficacy of LLLT in TMDs is primarily because of the variability in the laser dose. In our systematic review, laser dosage ranged between $1.5 \mathrm{~J} / \mathrm{cm} 2$ to $112.5 \mathrm{~J} / \mathrm{cm} 2$, except for 5 studies where data was not available (Table 1). Laser power ranged between $1.76 \mathrm{Mw}$ [28] to $500 \mathrm{~mW}$ [35].

The included RCTs also showed a wide disparity in the frequency of laser application, the number of sessions/weeks, and the total number of laser sessions. The studies showed that the number of sessions per week ranged from 1-7. Most of the studies argued for 2 sessions per week $[5,11,13,14,26,28,32,35,37,43,45,49-51,56,57]$. However, there was no mention of the number of sessions/ weeks in a few studies [25-28, 30, 34, 47]. The total number of laser applications also showed great variance, ranging from 4 to 20 sessions. Eight studies argued for a total of 8 sessions [5, 32, 43, 49-51, 56, 57], followed by 12 sessions in by 7 studies [10-12, 24, 36, $38,46]$, and 10 sessions in 6 studies [15, 26, 29, 31, 44, 45]. However, few studies provided no information on the total number of laser sessions $[25,27,30,35,47,48]$. The time of laser application also varied widely in the included studies. 


\section{JOURNAL of MEDICINE and LIFE}

Kulekcioglu et al. recommended using LLLT as an alternative to other conventional treatment modalities in TMD of myogenic and arthrogenic origin [58]. However, Machado et al. suggested that combination therapy of LLLT and oral motor exercises are more efficient for the rehabilitation of TMD patients [59]. Studies using supplementary diagnostic aids - panoramic radiography (OPG), computed tomography $(\mathrm{CT})$, and magnetic resonance imaging $(\mathrm{MRI})$ - should be vigilantly evaluated, as the interpretations of these investigations may not always correspond with the signs and symptoms of TMDs [53].

Few studies in our review used auxiliary diagnostic methods for TMD diagnosis. TMJ imaging using CT and MRI was done in a study by Shirani et al. [28], and OPG was used in studies conducted by Shobha et al. [5], Venancio et al. [14], Venezian et al. [32], and Carrasco et al. [51]. Over the last few years, LLLT has evolved as an excellent intervention for TMDs, owing to its analgesic, anti-inflammatory, and regenerative effects with no documented unfavorable outcomes and exceptional patient compliance. However, there is still no conclusive validation to substantiate or contradict LLLT for TMDs. Here, we have attempted to upgrade the clinical validation for LLLT effects on TMDs [4]. The strengths of our systematic review were the large number of included RCTs, hence a larger sample size that was analyzed. Regarding the limitations of the review, published literature on the use of LLLT in TMDs has revealed contradictory outcomes, primarily due to the variation in laser dosage [19].

The primary limitation of this systematic review was that only two specific databases were searched (PubMed and Science Direct) due to limited access to databases. This study advocated performing another systematic review with meta-analyses by incorporating some more databases to strengthen the findings. The disparity in the treatment parameters (dosage, power, wavelength, number, and frequency of laser application) and within the patient sample are the other limiting factor of this review. Generally, LLLT yields better efficacy when used within the electromagnetic radiation spectrum, incorporating higher irradiation parameters (higher dose and power), a greater number of sessions, and frequency of applications [53].

\section{CONCLUSION}

This systematic review aimed to re-validate the efficiency of LLLT in TMDs by thoroughly evaluating the previously conducted researches and further compare with placebo and other interventions. The study outcomes are expected to provide useful guidelines for practitioners treating patients with TMDs. The results demonstrate that LLLT appears to be efficient in diminishing TMD pain with variable effects on the outcome of secondary parameters. Also, LLLT provides advantages as the therapeutic regimen is non-invasive, reversible, with fewer adverse effects, and may also improve the psychological and emotional aspects associated with TMDs. Therefore, this systematic review highlights the role of LLLT as a promising therapeutic regimen for TMDs.

\section{ACKNOWLEDGMENTS}

\section{Conflict of interest}

The authors declare that there is no conflict of interest.

\section{REFERENCES}

1. Murphy MK, MacBarb RF, Wong ME, Athanasiou KA Temporomandibular disorders: a review of etiology, clinical management, and tissue engineering strategies. Int J Oral Maxillofac Implants. 2013;28[6]:393-414

2. Ouanounou A, Goldberg M, Haas DA. Pharmacotherapy in Temporomandibular Disorders: A Review. J Can Dent Assoc. 2017; 83:1-8.

3. Khairnar S, Bhate K, S N SK, Kshirsagar K, Jagtap B, Kakodkar P. Comparative evaluation of low-level laser therapy and ultrasound heat therapy in reducing temporomandibular joint disorder pain. J Dent Anesth Pain Med. 2017;19[5]:289-294.

4. Xu GZ, Jia J, Jin L, Li JH, Wang ZY, Cao DY. Low-Level Laser Therapy for Temporomandibular Disorders: A Systematic Review with Meta-Analysis. Pain Res Manag 2018:4230583

5. Shobha R, Narayanan VS, Jagadish Pai B S, Jaishankar H P, Jijin M J. Low-level laser therapy: A novel therapeutic approach to temporomandibular disorder - A randomized double-blinded, placebo-controlled trial. Indian J Dent Res. 2017; 28:380-387.

6. Alzarea BK. Temporomandibular Disorders [TMD] in Edentulous Patients: A Review and Proposed Classification [Dr. Bader's Classification]. J Clin Diagn Res. 2015;9[4]:6-9.
7. Romero-Reyes M, Uyanik JM. Orofacial pain management: current perspectives. J Pain Res. 2014; 7:99-115.

8. Abouelhuda AM, Khalifa AK, Kim YK, Hegazy SA. Non-invasive different modalities of treatment for temporomandibular disorders: review of literature. J Korean Assoc Oral Maxillofac Surg. 2018;244[2]:43-51.

9. Carli BM, Magro AK, Souza-Silva BN, Matoo FD, Carli JP, Paranhos LR, et al. The effect of Laser and Botulinum toxin in the treatment of Myofascial pain and Mouth Opening: Journal of Photochemistry \& Photobiology, B: Biology 2016;159:120-123.

10. Ahrari F, Madani AS, Ghafouri ZS, TunérJ. The Efficacy of Low-Level Laser Therapy for the Treatment of Myogenous Temporomandibular Joint Disorder. Lasers Med Sci 2014;29[2]:551-557.

11. Chellappa D, Thirupathy M. Comparative efficacy of low-level laser and TENS in the symptomatic relief of temporomandibular joint disorders: A randomized clinica trial. Indian J Dent Res 2020; 31:42-47.

12. Ferreira LA, de Oliveira RG, Guimarães JP, Carvalho AC, De Paula MV Laser acupuncture in patients with temporomandibular dysfunction: a randomized controlled trial. Lasers Med Sci. 2013;28[6]:1549-1558.

13. Emshoff R., Bosch R., Pumpel E., Schoning H., Strobl H Low-level laser therapy for treatment of temporomandibular joint pain: a double-blind and placebo-controlled trial.
Oral Surg Oral Med Oral Pathol Oral Radiol Endod. 2008;105[4]:452-456.

14. Venancio A., Camparis C. M., Lizarelli F. Low intensity laser therapy in the treatment of temporomandibular disorders: a double-blind study. J Oral Rehabil. 2010;32[11]:800-807.

15. Marini I, Gatto MR, Bonetti GA. Effects of super pulsed low-level laser therapy on temporomandibular joint pain. Clin J Pain. 2010;26[7]:611-616.

16. Wang X, Yang Z, Zhang W, Yi X, Liang C, Li X. Efficacy evaluation of low-level laser therapy on temporomandibular disorder. Cranio. 2011;29 [4]:393-395.

17. Chang WD, Lee CL, Lin HY, Hsu YC, Wang CJ, Lai PT. A meta-analysis of clinical effects of low-level laser therapy on temporomandibular joint pain. J Phys Ther Sci. 2014;26[8]:1297-1300.

18. Petrucci A, Sgolastra F, Gatto R, Mattei A, Monaco A. Effectiveness of low-level laser therapy in temporomandibular disorders: a systematic review and meta-analysis. J Orofac Pain. 2011; 25:298-307.

19. Shukla D, Muthusekhar MR. Efficacy of low-level laser therapy in temporomandibular disorders: a systematic review. Natl J Maxillofac Surg. 2016;7[1]:62-66 


\section{JOURNAL of MEDICINE and LIFE}

20. Melis M., Di Giosia M., Zawawi K. H. Low level laser therapy for the treatment of temporomandibular disorders: a systematic review of the literature. Cranio. 2012;30[4]:304-312

21. Gam AN, Thorsen H, Lonnberg F. The effect of low-leve laser therapy on musculoskeletal pain: a meta-analysis. Pain 1993;52[1]:63-66.

22. McNeely ML, Armijo Olivo S, Magee DJ. A systematic review of the effectiveness of physical therapy interventions for temporomandibular disorders.

Phys Ther 2006;86[5]:710-725

23. Chen J, Huang Z, Ge M, Gao M. Efficacy of low-level laser therapy in the treatment of TMDs: a meta-analysis of 14 randomised controlled trials. J Oral Rehabil. 2015;42[4]:291-299.

24. Brochado FT, Jesus LH, Carrard VC, Freddo AL, Chaves KD, Martins MD. Comparative effectiveness of photo biomodulation and manual therapy alone or combined in TMD patients: a randomized clinical trial. Braz Oral Res. 2018; $32: 50$

25. Altindiş $T$, Güngörmüş $M$. Thermographic evaluation of occlusal splint and low-level laser therapy in myofascial pain syndrome. Complement Ther Med. 2019; 44:277-281.

26. Madani AS, Ahrari F, Fallahrastegar A, Daghestani N. A Randomized Clinical Trial Comparing the Efficacy of Low-Level Laser Therapy [LLLT] and Laser Acupuncture Therapy [LAT] in Patients with Temporomandibular Disorders. Lasers Med Sci 2020;35[1]:181-192.

27. Rodrigues MF, Rodrigues ML, Bueno KS, Aroca JP, Camilotti V, Busato MC, et al. Effects of Low-Power Laser Auriculotherapy on the Physical and Emotional Aspects in Patients with Temporomandibular Disorders: A Blind, Randomized, Controlled Clinical Trial. Complement Ther Med. 2019; 42:340-346.

28. Shirani AM, Gutknecht N, Taghizadeh M, Mir M. Low-level laser therapy and myofacial pain dysfunction syndrome: a randomized controlled clinical trial. Lasers Med Sci. 2009;24[5]:715-720.

29. Demirkol N, Usumez A, Demirkol M, Sari F, Akcaboy C. Efficacy of low-level laser therapy in subjective tinnitus patients with temporomandibular disorders. Photomed Laser Surg 2017;35[8]:427-431.

30. Silva-Pereira TL, Flecha OD, Guimara RC, Douglas de Oliveira D, Adriana Maria Botelho AM,

Ramos-Gloria JC et al. Efficacy of red and infrared lasers in treatment of temporomandibular disorders-A double blind, randomized, parallel clinical trial. Cranio: The Journal of Craniomandibular \& Sleep Practice 2014;32[1]:51-56.

31. Demirkol N, Sari F, Bulbul M, Demirkol M, Simsek I, Usumez A. Effectiveness of occlusal splints and low-level laser therapy on myofascial pain. Lasers Med Sci. 2014;30[3]:1007-1012.

32. Venezian GC, da Silva MA, Mazzetto RG, Mazzetto MO. Low level laser effects on pain to palpation and electromyographic activity in TMD patients: a double-blind, randomized, placebo-controlled study. Cranio:The Journal of Craniomandibular \& Sleep Practice 2010;28[2]:84-91.

33. Cunha L. A, Firoozmand LM, Silva AP, Camargo SE, Esteves SA, Oliveira W. Efficacy of low-level laser therapy in the treatment of temporomandibular disorder. Int Dent J. 2008;58[4]:213-217.
34. Uemoto L, Garcia MA, Gouvêa CV, Vilella OV, Alfaya TA. Laser therapy and needling in myofascial trigger point deactivation. J Oral Sci. 2013;55[2]:175-181.

35. Öz S, Gökçen-Röhlig B, Saruhanoglu A, Tuncer EB. Management of myofascial pain: Low-level laser therapy versus occlusal splints. J Craniofac Surg. 2010; 21:1722-1728.

36. Cavalcanti MF, Silva UH, Leal-Junior EC, Lopes-Martins RA, Marcos LR, Pallotta RC, et al. Comparative study of the physiotherapeutic and drug protocol and low-level laser irradiation in the treatment of pain associated with temporomandibular dysfunction. Photomed Laser Surg 2016:34[12]:652-666.

37. Carli ML, Guerra MB, Nunes TB, di Matteo RC, de Luca CEP, Aranha AC, et al. Piroxicam and laser phototherapy in the treatment of TMJ arthralgia: a double-blind randomized controlled trial. J Oral Rehabil. 2013;40[3]:171-178.

38. Fornaini C, Pelosi A, Queirolo V, Vescovi P, Merigo E The "at-home LLLT" in temporo-mandibular disorders pain control: a pilot study. Laser Ther. 2015;24[1]:47-52.

39. Badel T, Pavičin IS, Čimić S, Zadravec D. Diagnostics and Management of Temporomandibular Joint Disorder - A Reported Case with a Review of Literature. J Dent Probl Solut 2016;3[1]:18-23.

40. Reneker J, Paz J, Petrosino C, Cook C. Diagnostic accuracy of clinical tests and signs of temporomandibular joint disorders: a systematic review of the literature. J Orthop Sports Phys Ther. 2011;41[6]:408-416.

41. Gil-Martínez A, Paris-Alemany A, López-de-UraldeVillanueva I, La Touche R. Management of pain in patients with temporomandibular disorder (TMD): challenges and solutions. J Pain Res. 2018; 11:571-587.

42. Rodrigues CA, Melchior MO, Magri LV, Mestriner WR, Mazzetto MO. Is the Masticatory Function Changed in Patients with Temporomandibular Disorders? Brazilian Dental Journal 2015;26[2]:181-185.

43. Mazzetto MO, Hotta TH, Pizzo RC. Measurements of jaw movements and TMJ pain intensity in patients treated with GaAlAs laser. Braz Dent J. 2010;21 [4]:356-360.

44. Röhlig BG, Kipirdi S, Meriç U, Çapan N, Keskin H Masticatory muscle pain and low-level laser therapy: a double-blind and placebo-controlled study. Turkiye Fiziksel Tip ve Rehabilitasyon Dergisi 2011; 57:31-37.

45.Silva MA, Botelho AL, Turim CV, Silva AM. Low level laser therapy as an adjunctive technique in the management of temporomandibular disorders. Cranio. 2013;30[4]:264-271.

46. Sancakli E, Gokcen-Rohlig B, Balik A, Ongul D, Kipird $\mathrm{S}$, Keskin H. Early results of low-level laser application for masticatory muscle pain: a double-blind randomized clinica study. BMC Oral Health 2015;15[1]:131

47. Pinho-Costa SA, Florezi GP, Artes GE, da Costa JR, Gallo RT, de Freista PM, et al. The analgesic effect of photobiomodulation therapy $[830 \mathrm{~nm}]$ on the masticatory muscles: a randomized, double-blind study. Braz. Oral Res 2017; 31:107

48. De Godoy CHL. Effect of Low-Level Laser Therapy on Adolescents with Temporomandibular Disorder: A Blind Randomized Controlled Pilot Study. Journ of Oral \& maxillofac surg 2015;73[4]:622-629.

49. Maia ML, Ribeiro MG, Maia LG, Martins Maia LG, Stuginski-Barbosa J, Costa YM, et al. Evaluation of low-leve laser therapy effectiveness on the pain and masticatory performance of patients with myofascial pain. Lasers Med Sci. 014;29[1]:29-35.

50. Magri LV, Carvalho VA, Rodrigues FC, Bataglion C. Effectiveness of low-level laser therapy on pain intensity Effectiveness of low-level laser therapy on pain intensity,
pressure pain threshold, and SF-MPQ indexes of women with myofascial pain. Lasers Med Sci 2017;32[2]:419-428.

51. Carrasco TG, Mazzetto MO, Mazzetto RG, Mestrine WJ. Low intensity laser therapy in temporomandibular disorder: a phase II double-blind study. Cranio 2008;26[4]:274-281.

52. Chisnoiu AM, Picos AM, Popa S, Daniel-Chisnoiu P Lascu L, Picos A, et al. Factors involved in the etiology of temporomandibular disorders - a literature review. Cluju Med. 2015;88[4]:473-478.

53. Maia ML, Bonjardim LR, Quintans S, Ribeiro MA, Maia LG, Conti PC. Effect of low-level laser therapy on pain levels in patients with temporomandibular disorders: a systematic review. Journal of applied oral science: revista FOB 2012;20[6]:594-602.

54. Zokaee H, Akbari Zahmati AH, Mojrian N, Boostani A, Vaghari M. Efficacy of low-level laser therapy on orofacial pain: A literature review. Adv Hum Biol 2008; 8:70-73.

55. Bjordal JM., Couppé C, Chow RT, Tunér J, Ljunggren EA. A systematic review of low-level laser therapy with location-specific doses for pain from chronic joint disorders Aust J Physiotherapy 2003;49[2]:107-16.

56. Frare J, Nicolau R. Clinical analysis of the effect of laser photo biomodulation [GaAs-904 nm] on temporomandibular joint dysfunction. Rev Bras Fisioter. 2008;12[1]:37-42.

57. Mazzetto M. O., Carrasco T. G., Bidinelo E. F., Andrade Pizzo R. C., Mazzetto R. G. Low intensity laser application in temporomandibular disorders: a phase I double-blind study. Cranio. 2007;25[3]:186-192.

58. Kulekcioglu S., Sivrioglu K., Ozcan O., Parlak M. Effectiveness of low-level laser therapy in temporomandibular disorder. Scand J Rheumatol. 2003;32[2]:1 14-118.

59. Machado BCZ, Mazzetto MO, Silva M, Felicio CM. Effects of oral motor exercises and laser therapy on chronic temporomandibular disorders: a randomized study with follow-up. Lasers Med Sci. 2016;31[5]:945-954. 